\title{
Emission inventory of air pollutants and chemical speciation for specific anthropogenic sources based on local measurements in the Yangtze River Delta region, China
}

Jingyu An et al.

Correspondence to: Cheng Huang (huangc@saes.sh.cn)

The copyright of individual parts of the supplement might differ from the CC BY 4.0 License. 


\section{Sources classification}

Table S1 shows the source classification in different levels in the Yangtze River Delta (YRD) region. In the first level, the sources are classified into ten categories, including stationary combustion source, industrial process source, industrial solventuse source, mobile source, dust source, oil storage and transportation source, residential source, waste treatment and disposal source, and agricultural source. The second-level categories of stationary combustion source include power plants and boilers. The threelevel classification is based on fuel type, which mainly includes coal, natural gas, fuel oil, diesel, and biomass, etc. The fourth level is classified according to the combustion method, such as grate-fired boilers, pulverized coal boilers, and fluidized bed combustion (FBC) boilers, etc. Industrial process and solvent-use sources are divided into second- and third-level according to their industrial sectors and product or material types. Some sources are further subdivided into the fourth-level based on their detailed product types or emission segments. For example, in the coating production industry, solvent- and water-based coatings are further separated. In the ironmaking industry, organized emission segments including sintering and blast furnaces, and fugitive emission segments are further separated. The second-level classification of mobile source includes motor vehicles, ships, non-road machinery, and civil aviation aircraft. The third-level is divided according to their detailed types. Motor vehicles include lightduty passenger cars, light-duty trucks, heavy-duty passenger vehicles, heavy-duty trucks, and motorcycles, etc. Non-road machinery is divided into construction machinery, port machinery, factory machinery, agricultural machinery, and airport ground handling equipment (GHE) according to their purpose. The fourth-level classification mainly considers their fuel type. The motor vehicles and non-road machinery are further classified into the fifth-level according to their emission levels.

2 The dust source is mainly divided into two categories: construction dust and road dust.

3 The oil storage and transportation sources mainly include oil and gas volatilization 4 sources such as gas stations, storage tanks and loading and unloading operations. The 
residential source includes residential combustion, residential solvent-use, catering, and human excretion. The residential solvent-use source is further divided into architectural coatings, consumer products, auto repair, dry cleaning, and hospital solvent-use. The waste treatment and disposal sources include sewage treatment and waste treatment. The waste treatment source is further divided into landfill and compost, while the incineration is included in the boiler source. The agricultural source includes livestock and poultry breeding, $\mathrm{N}$-fertilizer application, and biomass burning. The livestock and poultry breeding and biomass burning sources are further divided into the third-level based on the species of livestock and poultry and crop residue types, respectively. Biomass burning includes two categories of field burning and household stoves, and then the fourth level according to different straw types, such as soybean, rape, rice, wheat, corn, etc.

\section{Activity data sources}

Table S2 shows the emission estimation methods and activity data sources for various sources and their reliability levels. The activity data for stationary combustion source, industrial process source and industrial solvent-use source are mainly derived from 2017 environmental statistics, covering nearly 30,000 major point sources in the YRD region. The data used in this study mainly includes fuel consumption, sulfur and ash content, product output, raw material consumption, solvent usage, and pollutant removal method and its efficiency, etc. However, the environmental statistics cannot include all industrial sources. The difference between the total fuel consumption and product output in the statistical yearbook and the sum of environmental statistics for each city is taken as an area source.

For motor vehicles, the number of various vehicle types come from the statistical yearbook of each city. To prepare the fleet composition data, detailed information about vehicle technology, fuel type, emission standard, and vehicle age was surveyed in the representative cities like Nanjing, Hangzhou, and Shanghai. The annual average VMT of each vehicle type is simultaneously obtained from local survey in these cities. The 
total VMT is then allocated into four road types, including highways, elevated roads, arterial roads, and residential roads, based on their traffic flows and road length. The average speeds and driving cycles for different road types were obtained from previous studies (Huang et al., 2015; Tang et al., 2018). Since there are no ready-made statistics for other machines besides agricultural machinery, the numbers of non-road machinery are calculated based on local survey data from Shanghai and Hangzhou. The number of construction machinery is calculated by the construction area; the number of port machinery is calculated according to the cargo throughput for each port; the number of factory machinery is estimated according to the total output value of the heavy industry; the number of GHE is estimated according to the passenger volume of the civil aviation. The working hours of various non-road machinery types come from local survey results in Shanghai and Hangzhou (Lu et al., 2017). The activity data of ships come from the AIS data for the East China Sea in 2017. The number of takeoffs and landings of civil aviation aircrafts at each airport are obtained based on the flight information survey on the Internet. The engine type for each aircraft can be determined based on the engine database released by ICAO.

The activity data of other sources is mainly from statistical yearbooks. The construction dust emissions are estimated based on the construction area of each city. The dust control measures during construction period are from the surveys of typical cities such as Hangzhou and Shanghai. The activity data of road dust is basically the same as that of motor vehicle emissions. NMVOC emissions from oil and gas storage and transportation sources are related with the transfer of oil products from various cities and the consumption of gas stations. All of the storage tanks and gas stations have been equipped with oil and gas recovery facilities. The activity data of residential combustion sources are derived from fuel statistics including coal, natural gas (NG), and liquefied petroleum gas (LPG), etc., used by residents of various cities. The activity data for architectural coatings is calculated based on the newly built and built-up housing area of each city, combined with the solvent consumption used per unit area 
111 from survey. The activity data for catering, human excretion, consumer products, auto

112 repair, and waste treatment and disposal sources are derived from statistics on 113 population, vehicle ownership, and sewage and solid waste disposal. The activity data

114 of agricultural sources comes from the statistics livestock and poultry farming in

115 different species and nitrogen fertilizer application in each city. The activity data of

116 Biomass burning is estimated based on the yield of different crops multiplied by the 117 ratio of straw and then minus the recovery of straw.

118

\section{Emission factors determination}

Table S1 lists the emission factors (EFs) for various emission sources and their references. The EFs of coal-fired power plants mainly come from domestic measurement results (Liu, 2008; Yao et al., 2009; Zhao et al., 2010; Wang et al., 2011; Sun, 2015). The EFs of coal-fired boilers are determined by our local measurements in the YRD region (Lou, 2014; Xu et al., 2018). For other fuels and non-criteria pollutants (such as NMVOCs, $\mathrm{NH}_{3}$, etc.), the EFs mainly refer to the USEPA's AP-42 (USEPA, 2002) and European's EMEP datasets (EEA, 2013). The EFs for industrial process sources are mainly derived from the datasets provided by AP-42, EMEP and domestic emission inventory (EI) guidebook of China (MEP, 2014). VOC content of coatings, inks and adhesives from industrial solvent-use sources are determined by local surveys in Shanghai and Hangzhou.

The EFs of motor vehicles are calculated by the International Vehicle Emissions (IVE) model and then corrected based on the local measurements of 102 light-duty gasoline vehicles (LDGVs) with Euro 2-5 standards and 33 heavy-duty diesel vehicles (HDDVs) with Euro 3-5 standards (Huang et al., 2016; Huang et al., 2017; Huang et al., 2018a; Huang et al., 2018b). The EFs for non-road machinery are referenced from the NONROAD model (USEPA, 2010) and domestic EI guidebook of China (MEP, 2014), and then corrected based on local measurements in previous studies in China (Fu et al., 2012; Fu et al., 2013; Ge et al., 2013; Qu et al., 2015; Li et al., 2016). The $\mathrm{NO}_{x}$ EFs from local measurements are generally higher than those recommended by the 
model and guidebook, while other pollutants are relatively close. The EFs of ships are

140 strongly related with the quality of fuel oils. China has executed the legislation that

141 requires ships to use LSF $(<0.5 \mathrm{wt} \% \mathrm{~S})$ when at berth beginning from January 1, 2017.

142 Therefore, the EFs were corrected based on the fuel quality in this study. Detailed

143 information is provided in a previous study (Fan et al., 2016). The $\mathrm{NO}_{x}, \mathrm{CO}$, and

144 NMVOC EFs of civil aircraft engines in each mode of operation are taken from the

145 Aircraft Engine Emissions Database from ICAO (ICAO, 2014). The $\mathrm{SO}_{2}$ and PM

146 emissions are estimated using the mass balance and FOA3.0 methods, respectively.

147 The EFs of dust sources are mainly derived from the EI guidebook of China (MEP,

148 2014). Those of oil storage and transportation sources are determined from local

149 surveys of the storage and transportation process of oil depots and gas stations. A total

150 of 294 storage tanks for different oil types and more than 400 gas stations were surveyed

151 in Shanghai and Hangzhou. Then the NMVOC EFs for per unit turnover for the storage

152 and transportation process of oil depots and gas stations were determined based on the

153 method recommended by USEPA (USEPA, 2015). Besides of the oil storage and

154 transportation sources, the EFs of residential solvent-use sources, like architectural

155 coatings, auto repair, dry cleaning, and hospital solvent-use, are also estimated based

156 on the local surveys of typical sources in the YRD region. The EFs of catering and

157 biomass burning are both determined based on the local measurements (Tang et al.,

158 2014; Wang et al., 2018; Gao et al., 2019). For agricultural sources, the $\mathrm{NH}_{3}$ emission

159 factors of swine and dairy cow breeding, and fertilization in paddy and wheat fields are

160 localized base on the measurements in Shanghai (Chen, 2017a; Chen et al., 2017b; Xia

161 et al., 2018; Zhou, 2019). For the other area sources, the EFs are mainly referred to the

162 EI guidebook of China (MEP, 2014).

\section{4. PM2.5 and NMVOC speciation}

$164 \mathrm{PM}_{2.5}$ and NMVOC speciation profiles of each source were determined based on 165 local measurements in this and previous studies and the USEPA's SPECIATE4.4 166 database (Hsu et al., 2014). Table S3 shows the references, samples, and sampling and 
analytical methods of different sources in this study. A total of $34 \mathrm{PM}_{2.5}$ sources and 64 NMVOC sources were localized in the YRD region. The speciation of $\mathrm{PM}_{2.5}$ included carbonaceous, ionic, and elemental components, which were analyzed by a thermal/optical carbon analyzer (Model 2001, DRI, USA), an ion chromatography (Model 940, Metrohm, Switzerland), and an energy-dispersive X-ray fluorescence spectrometer (ED-XRF, Epsilon 5, PANalytical, Finland) or an inductively coupled plasma direct reading spectroscopy (ICP-AES, JOBIN-YVON, France), respectively. VOC species were analyzed by a gas chromatograph with a mass spectrometer and flame ionization detector (GC-MS/FID, TH_PKU-300, China).

\section{Emissions for different cities and sources}

Annual air pollutant emissions from each city in the YRD region is shown in Table S4. $\mathrm{SO}_{2}$ emissions mainly came from Suzhou, Xuzhou and Changzhou in Jiangsu Province. The cities with the largest $\mathrm{NO}_{x}$ emissions were Shanghai, Suzhou and Xuzhou, followed by Ningbo and Hangzhou in Zhejiang Province. CO emissions from Suzhou and Nanjing were relatively higher, followed by Shanghai. VOC emissions came from Shanghai, Suzhou and Ningbo in turn. Xuzhou in Jiangsu Province had the largest PM $\mathrm{M}_{10}$ and $\mathrm{PM}_{2.5}$ emissions, followed by Hefei in Anhui Province. $\mathrm{NH}_{3}$ emission distribution was quite different from the pollutants mentioned above. Yancheng, Xuzhou, and Nantong in Jiangsu Province had the largest emissions, followed by Fuyang and Suzhou in Anhui Province. Table S5 shows the air pollutant emissions from 10 major source categories and 55 subcategories. Among them, industrial process and solvent-use sources include 13 and 12 major sectors, respectively. The mobile sources include 14 categories, including 7 types of vehicles and 5 types of non-road machinery.

\section{Model evaluation}

We used the Community Modeling and Analysis System (CMAQ version 5.3) to simulate the concentrations of $\mathrm{SO}_{2}, \mathrm{NO}_{2}, \mathrm{PM}_{2.5}, \mathrm{PM}_{10}, \mathrm{O}_{3}$, and $\mathrm{CO}$ in the $\mathrm{YRD}$ region in January and July 2017, then compared with the observation data in each city of the region. Figure S2 shows the model domain and the locations of observation data for 
meteorology and $\mathrm{PM}_{2.5}$ chemical composition. Simulations were conducted for three nested grids with horizontal resolution of $36 \mathrm{~km}(\mathrm{D} 1), 12 \mathrm{~km}$ (D2) and $4 \mathrm{~km}(\mathrm{D} 3)$, respectively. D1 covers most of China and the surrounding countries including Japan and South Korea; D2 covers eastern China and D3 covers the entire YRD region and its surrounding land and waters. Meteorological fields were provided by the Weather Research and Forecasting (WRF version 3.7) model with 27 vertical layers extending to the tropopause (100hpa). WRF initial and boundary conditions (ICs, BCs) are based on the $1^{\circ} \times 1^{\circ}$ reanalysis data from the National Centers for Environmental Prediction Final Analysis (NCEP-FNL). Physical options used in the WRF simulation are listed in Table S1. The emission inventory developed in this study was used to produce the emission system in the YRD region while emission beyond YRD was supplied by Multiresolution Emission Inventory for China (MEIC-2016) (http://www. meicmodel.org) and the Model Inter-Comparison Study (MIX) emission inventory for 2010 (Li et al., 2017). Biogenic emissions are estimated by Model of Emissions of Gases and Aerosols from Nature (MEGAN) v2.1 using WRF meteorology predictions. The Sparse Matrix Operator Kernel Emissions (SMOKE, https://www.cmascenter.org/smoke) model is applied to process emissions for input to CMAQ. CMAQ version 5.3.0 (https://www.cmascenter.org/cmaq/) was used to simulate atmospheric pollutants concentrations. ICs and BCs of D1 domain are based on a Model For Ozone And Related Chemical Tracers (MOZART) global simulation (https://www.acom.ucar.edu/wrf-chem/mozart.shtml). For the inner D2 and D3 domain, ICs and $\mathrm{BCs}$ are extracted from the simulation results of the outer domains. Options selected for the CMAQ simulations include the CB6r3 gas phase chemistry, the AERO6 aerosol scheme, the Regional Acid Deposition Model (RADM) model aqueous phase chemistry, ISORROPIA inorganic particulate thermodynamics, and SOAP secondary organic aerosol scheme.

Table S7 compares the average concentration of observation and simulation data in each city of the YRD region in January and July 2017 and shows the statistical results 
223 of model performance. We used Normalized Mean Bias (NMB), Normalized Mean 224 Error (NME), Mean Fractional Bias (MFB) and Mean Fractional Error (MFE) to 225 characterize the deviation of simulation concentrations from measured results. For 226 NMB and MFB, negative numbers indicate that the simulation result is lower, while 227 positive numbers are the opposite. The smaller the absolute value of these four statistics, 228 the higher the accuracy.

229

230

231

232 
233 Table S1. Source classification and emission factors.






\begin{tabular}{|c|c|c|c|c|c|c|c|c|c|c|c|c|}
\hline \multicolumn{4}{|c|}{ Source classification } & \multirow{2}{*}{ Units } & \multicolumn{7}{|c|}{ Emission factors } & \multirow{2}{*}{ References } \\
\hline 1st level & 2nd level & 3rd level & 4th level & & $\mathrm{SO}_{2}$ & $\mathrm{NO}_{x}$ & $\mathrm{CO}$ & NMVOCs & $\mathrm{PM}_{10}$ & $\mathrm{PM}_{2.5}$ & $\mathrm{NH}_{3}$ & \\
\hline & & Sugar production & - & $\mathrm{g} \cdot \mathrm{kg}^{-1}$-products & - & - & - & 0.20 & 0.52 & 0.26 & - & EEA, 2013 \\
\hline & & Meat processing & - & $\mathrm{g} \cdot \mathrm{kg}^{-1}$-products & - & - & - & 0.52 & - & - & - & EEA, 2013 \\
\hline & & Nut processing & - & $\mathrm{g} \cdot \mathrm{kg}^{-1}$-products & - & - & - & - & 0.53 & 0.26 & - & USEPA, 2002 \\
\hline & Food manufacturing & Baked goods manufacturing & Bread & $\mathrm{g} \cdot \mathrm{kg}^{-1}$-products & - & - & - & 4.71 & - & - & - & EEA, 2013 \\
\hline & & & Biscuits & $\mathrm{g} \cdot \mathrm{kg}^{-1}$-products & - & - & - & 1.00 & - & - & - & MEP, 2014 \\
\hline & Beverage manufacturing & Alcohol & - & $\mathrm{g} \cdot \mathrm{kg}^{-1}$-products & - & - & - & 32.11 & - & - & - & USEPA, 2002 \\
\hline & & Liquor & - & $\mathrm{g} \cdot \mathrm{kg}^{-1}$-products & - & - & - & 14.45 & - & - & - & USEPA, 2002 \\
\hline & & Red wine & - & $\mathrm{g} \cdot \mathrm{kg}^{-1}$-products & - & - & - & 3.85 & - & - & - & USEPA, 2002 \\
\hline & & Beer & - & $\mathrm{g} \cdot \mathrm{kg}^{-1}$-products & - & - & - & 0.25 & - & - & - & MEP, 2014 \\
\hline & Tobacco manufacturing & Tobacco & - & $\mathrm{g} \cdot \mathrm{kg}^{-1}$-products & - & - & - & 0.17 & - & - & - & USEPA, 2002 \\
\hline & Wood processing & Plywood & - & $\mathrm{g} \cdot \mathrm{m}^{-3}$-products & - & - & - & 0.25 & 0.03 & 0.02 & - & USEPA, 2002 \\
\hline & & Oriented strand board & - & $\mathrm{g} \cdot \mathrm{kg}^{-1}$-products & - & - & - & 0.26 & 0.04 & 0.02 & - & USEPA, 2002 \\
\hline & & Particleboard & - & $\mathrm{g} \cdot \mathrm{kg}^{-1}$-products & - & - & - & 0.92 & 0.05 & 0.02 & - & USEPA, 2002 \\
\hline & & Fiberboard & - & $\mathrm{g} \cdot \mathrm{kg}^{-1}$-products & - & - & - & 1.02 & 0.05 & 0.02 & - & USEPA, 2002 \\
\hline & & Other wood products & - & $\mathrm{g} \cdot \mathrm{kg}^{-1}$-products & - & - & - & 0.10 & 0.01 & 0.00 & - & USEPA, 2002 \\
\hline & Papermaking & Pulp manufacturing & - & $\mathrm{g} \cdot \mathrm{kg}^{-1}$-products & 11.38 & - & 5.55 & 0.66 & 3.14 & 2.44 & - & USEPA, 2002 \\
\hline & Petroleum refining & Process devices & - & $\mathrm{g} \cdot \mathrm{kg}^{-1}$-materials & - & - & - & 0.44 & - & - & - & Local surveys \\
\hline & & Equipment leak & - & $\mathrm{g} \cdot \mathrm{kg}^{-1}$-materials & - & - & - & 0.48 & - & - & - & Local surveys \\
\hline & & Storage tanks & - & $\mathrm{g} \cdot \mathrm{kg}^{-1}$-materials & - & - & - & 0.45 & - & - & - & Local surveys \\
\hline & & Bulk loading & - & $\mathrm{g} \cdot \mathrm{kg}^{-1}$-materials & - & - & - & 0.13 & - & - & - & Local surveys \\
\hline & & Flares & - & $\mathrm{g} \cdot \mathrm{kg}^{-1}$-materials & - & - & - & 0.001 & - & - & - & EEA, 2013 \\
\hline & & Wastewater treatment & - & $\mathrm{g} \cdot \mathrm{kg}^{-1}$-materials & - & - & - & 0.25 & - & - & - & Local surveys \\
\hline
\end{tabular}




\begin{tabular}{|c|c|c|c|c|c|c|c|c|c|c|c|c|}
\hline \multicolumn{4}{|c|}{ Source classification } & \multirow{2}{*}{ Units } & \multicolumn{7}{|c|}{ Emission factors } & \multirow{2}{*}{ References } \\
\hline 1st level & 2nd level & 3 rd level & 4th level & & $\mathrm{SO}_{2}$ & $\mathrm{NO}_{x}$ & $\mathrm{CO}$ & NMVOCs & $\mathrm{PM}_{10}$ & $\mathrm{PM}_{2.5}$ & $\mathrm{NH}_{3}$ & \\
\hline & & Cooling Tower & - & $\mathrm{g} \cdot \mathrm{kg}^{-1}$-materials & - & - & - & 0.02 & - & - & - & Local surveys \\
\hline & & Petrochemical furnace & Refinery dry gas & $\mathrm{g} \cdot \mathrm{kg}^{-1}$-fuel & - & 7.78 & 1.01 & 0.37 & 0.04 & 0.04 & - & USEPA, 2002 \\
\hline & Coking & Coke production & Coke oven & $\mathrm{g} \cdot \mathrm{kg}^{-1}$-materials & 1.47 & 0.82 & 0.34 & 0.05 & 0.14 & 0.14 & - & USEPA, 2002 \\
\hline & & & Fugitive emission & $\mathrm{g} \cdot \mathrm{kg}^{-1}$-materials & 0.12 & 0.01 & 15.04 & 0.04 & 2.94 & 1.86 & - & USEPA, 2002 \\
\hline & Chemical manufacturing & Organic chemical manufacturing & Overall & $\mathrm{g} \cdot \mathrm{kg}^{-1}$-products & - & - & - & 17.61 & - & - & - & USEPA, 2002 \\
\hline & & Inorganic chemical manufacturing & Sulfuric acid & $\mathrm{g} \cdot \mathrm{kg}^{-1}$-products & 15.28 & - & - & - & - & - & - & USEPA, 2002 \\
\hline & & & Nitric acid & $\mathrm{g} \cdot \mathrm{kg}^{-1}$-products & - & 4.71 & - & - & - & - & - & USEPA, 2002 \\
\hline & & & Calcium carbide & $\mathrm{g} \cdot \mathrm{kg}^{-1}$-materials & 3.00 & - & - & - & 5.82 & 2.91 & - & USEPA, 2002 \\
\hline & & Fertilizer & Synthetic ammonia & $\mathrm{g} \cdot \mathrm{kg}^{-1}$-products & 0.03 & - & 7.90 & 4.72 & - & - & 2.10 & USEPA, 2002 \\
\hline & & & Urea & $\mathrm{g} \cdot \mathrm{kg}^{-1}$-products & - & - & - & - & 1.31 & 0.81 & 10.51 & USEPA, 2002 \\
\hline & & & NPK Fertilizer & $\mathrm{g} \cdot \mathrm{kg}^{-1}$-products & - & - & - & - & 0.29 & 0.20 & 1.89 & EEA, 2013 \\
\hline & & Pesticide & - & $\mathrm{g} \cdot \mathrm{m}^{-3}$-products & - & - & - & 0.001 & - & - & - & USEPA, 2002 \\
\hline & & Coating production & Solvent-based coating & $\mathrm{g} \cdot \mathrm{kg}^{-1}$-products & - & - & - & 15.00 & - & - & - & USEPA, 2002 \\
\hline & & & Waterborne coating & $\mathrm{g} \cdot \mathrm{kg}^{-1}$-products & - & - & - & 3.00 & - & - & - & Local surveys \\
\hline & & & Varnish & $\mathrm{g} \cdot \mathrm{kg}^{-1}$-products & - & - & - & 46.25 & - & - & - & USEPA, 2002 \\
\hline & & Ink production & Oily ink & $\mathrm{g} \cdot \mathrm{kg}^{-1}$-products & - & - & - & 75.00 & - & - & - & USEPA, 2002 \\
\hline & & & Water-based ink & $\mathrm{g} \cdot \mathrm{kg}^{-1}$-products & - & - & - & 15.00 & - & - & - & Local surveys \\
\hline & & & Mineral ink & $\mathrm{g} \cdot \mathrm{kg}^{-1}$-products & - & - & - & 20.00 & - & - & - & USEPA, 2002 \\
\hline & & Synthetic resin & Overall & $\mathrm{g} \cdot \mathrm{kg}^{-1}$-products & - & - & - & 5.77 & - & - & - & USEPA, 2002; EEA, 2013 \\
\hline & & Synthetic rubber & Overall & $\mathrm{g} \cdot \mathrm{kg}^{-1}$-products & - & - & - & 4.18 & - & - & - & USEPA, 2002 \\
\hline & & Synthetic fiber monomer & Overall & $\mathrm{g} \cdot \mathrm{kg}^{-1}$-products & - & - & - & 48.79 & - & - & - & USEPA, 2002; MEP, 2014 \\
\hline & & Specialty chemicals & Adhesive & $\mathrm{g} \cdot \mathrm{kg}^{-1}$-products & - & - & - & 227.00 & - & - & - & USEPA, 2002 \\
\hline
\end{tabular}




\begin{tabular}{|c|c|c|c|c|c|c|c|c|c|c|c|c|}
\hline \multicolumn{4}{|c|}{ Source classification } & \multirow{2}{*}{ Units } & \multicolumn{7}{|c|}{ Emission factors } & \multirow{2}{*}{ References } \\
\hline 1st level & 2nd level & 3rd level & 4th level & & $\mathrm{SO}_{2}$ & $\mathrm{NO}_{x}$ & $\mathrm{CO}$ & NMVOCs & $\mathrm{PM}_{10}$ & $\mathrm{PM}_{2.5}$ & $\mathrm{NH}_{3}$ & \\
\hline & & & Accelerator & $\mathrm{g} \cdot \mathrm{kg}^{-1}$-products & - & - & - & 1.87 & - & - & - & USEPA, 2002 \\
\hline & & & Carbon black & $\mathrm{g} \cdot \mathrm{kg}^{-1}$-products & 7.13 & 1.08 & 720.49 & 26.20 & 1.31 & 0.65 & - & USEPA, 2002 \\
\hline & & & Charcoal & $\mathrm{g} \cdot \mathrm{kg}^{-1}$-products & - & 12.00 & 145.00 & 135.00 & 12.80 & 6.40 & - & USEPA, 2002 \\
\hline & & Explosive & Overall & $\mathrm{g} \cdot \mathrm{kg}^{-1}$-products & 22.94 & 30.58 & - & - & 0.72 & 0.36 & - & USEPA, 2002 \\
\hline & & Household chemicals & Active agent & $\mathrm{g} \cdot \mathrm{kg}^{-1}$-products & - & - & - & 0.98 & - & - & - & USEPA, 2002 \\
\hline & & & Spices & $\mathrm{g} \cdot \mathrm{kg}^{-1}$-products & - & - & - & 22.89 & - & - & - & Local surveys \\
\hline & & Chemical fiber & Synthetic fiber manufacturing & $\mathrm{g} \cdot \mathrm{kg}^{-1}$-products & - & - & - & 42.31 & - & - & - & USEPA, 2002 \\
\hline & & & Synthetic fiber processing & $\mathrm{g} \cdot \mathrm{kg}^{-1}$-products & - & - & - & 0.36 & - & - & - & USEPA, 2002 \\
\hline & Pharmaceutical manufacturing & Chemical drug & - & $\mathrm{g} \cdot \mathrm{kg}^{-1}$-products & - & - & - & 260.00 & - & - & - & MEP, 2014 \\
\hline & Rubber and plastic manufacturing & Rubber products & Tire & g. tire $^{-1}$ & - & - & - & 329.60 & - & - & - & USEPA, 2002 \\
\hline & & & General rubber products & $\mathrm{g} \cdot \mathrm{kg}^{-1}$-products & - & - & - & 6.64 & - & - & - & USEPA, 2002 \\
\hline & & Plastic products & General plastic products & $\mathrm{g} \cdot \mathrm{kg}^{-1}$-materials & - & - & - & 0.54 & - & - & - & USEPA, 2002 \\
\hline & & & Foam plastic & $\mathrm{g} \cdot \mathrm{kg}^{-1}$-products & - & - & - & 0.77 & - & - & - & MEP, 2014 \\
\hline & & & Poly urethane & $\mathrm{g} \cdot \mathrm{kg}^{-1}$-products & - & - & - & 147.00 & - & - & - & USEPA, 2002 \\
\hline & & & Polyvinyl chloride & $\mathrm{g} \cdot \mathrm{kg}^{-1}$-products & - & - & - & 10.20 & - & - & - & USEPA, 2002 \\
\hline & & & Plastic tape & $\mathrm{g} \cdot \mathrm{m}^{-2}$-products & - & - & - & 9.00 & - & - & - & USEPA, 2002 \\
\hline & Non-metallic mineral manufacturing & Cement manufacturing & Cement kiln & $\mathrm{g} \cdot \mathrm{kg}^{-1}$-products & 0.11 & 2.10 & 1.80 & 0.06 & 3.81 & 1.17 & - & USEPA, 2002 \\
\hline & & & Fugitive emission & $\mathrm{g} \cdot \mathrm{kg}^{-1}$-products & - & - & - & - & 0.24 & 0.05 & - & USEPA, 2002 \\
\hline & & Lime manufacturing & Lime kiln & $\mathrm{g} \cdot \mathrm{kg}^{-1}$-products & 2.70 & 1.60 & 0.74 & - & 22.00 & 2.52 & - & USEPA, 2002 \\
\hline & & & Fugitive emission & $\mathrm{g} \cdot \mathrm{kg}^{-1}$-products & - & - & - & - & 11.54 & 1.33 & - & USEPA, 2002 \\
\hline & & Plaster manufacturing & - & $\mathrm{g} \cdot \mathrm{kg}^{-1}$-products & - & - & - & - & 8.22 & 1.78 & - & USEPA, 2002 \\
\hline & & Gypsum board & - & $\mathrm{g} \cdot \mathrm{m}^{-2}$-products & - & - & - & - & 8.76 & 4.38 & - & USEPA, 2002 \\
\hline
\end{tabular}









\begin{tabular}{|c|c|c|c|c|c|c|c|c|c|c|c|c|}
\hline \multicolumn{4}{|c|}{ Source classification } & \multirow{2}{*}{ Units } & \multicolumn{7}{|c|}{ Emission factors } & \multirow{2}{*}{ References } \\
\hline \multirow[t]{10}{*}{ 1st level } & 2nd level & 3 rd level & 4th level & & $\mathrm{SO}_{2}$ & $\mathrm{NO}_{x}$ & $\mathrm{CO}$ & NMVOCs & $\mathrm{PM}_{10}$ & $\mathrm{PM}_{2.5}$ & $\mathrm{NH}_{3}$ & \\
\hline & & Primary lead & - & $\mathrm{g} \cdot \mathrm{kg}^{-1}$-products & - & - & - & - & 1.25 & 0.63 & - & EEA, 2013 \\
\hline & & Primary zinc & - & $\mathrm{g} \cdot \mathrm{kg}^{-1}$-products & - & - & - & - & 0.40 & 0.30 & - & EEA, 2013 \\
\hline & & Secondary copper & - & $\mathrm{g} \cdot \mathrm{kg}^{-1}$-materials & - & - & - & - & 60.41 & 30.20 & - & USEPA, 2002 \\
\hline & & Secondary aluminum & - & $\mathrm{g} \cdot \mathrm{kg}^{-1}$-products & - & - & - & - & 6.97 & 5.20 & - & USEPA, 2002 \\
\hline & & Secondary lead & - & $\mathrm{g} \cdot \mathrm{kg}^{-1}$-materials & - & - & - & - & 4.75 & 2.38 & - & USEPA, 2002 \\
\hline & & Secondary zinc & - & $\mathrm{g} \cdot \mathrm{kg}^{-1}$-products & - & - & - & - & 23.89 & 11.94 & - & USEPA, 2002 \\
\hline & \multirow[t]{3}{*}{ Other miscellaneous sources } & Explosives detonation & - & $\mathrm{g} \cdot \mathrm{kg}^{-1}$-materials & 1.00 & 17.00 & 114.11 & - & - & - & 12.43 & USEPA, 2002 \\
\hline & & Abrasive blasting & - & $\mathrm{g} \cdot \mathrm{kg}^{-1}$-products & - & - & - & - & 13.00 & 1.30 & - & USEPA, 2002 \\
\hline & & Electric arc welding & - & $\mathrm{g} \cdot \mathrm{kg}^{-1}$-materials & - & - & - & - & 12.46 & 6.23 & - & USEPA, 2002 \\
\hline Industrial solvent-use sources & - & - & - & - & \multicolumn{8}{|c|}{ Calculated based on solvent consumption } \\
\hline \multirow[t]{12}{*}{ Mobile sources } & Motor vehicles & Light-duty gasoline vehicles & Euro 0 & $\mathrm{~g} \cdot \mathrm{km}^{-1}$ & - & 2.80 & 28.68 & 6.53 & 0.12 & 0.12 & 0.00 & \multirow{6}{*}{$\begin{array}{l}\text { Local measurements (Huang et } \\
\text { al., 2017; Huang et al., 2018a; } \\
\text { Huang et al., 2018b) }\end{array}$} \\
\hline & & & Euro 1 & $\mathrm{~g} \cdot \mathrm{km}^{-1}$ & - & 1.23 & 13.52 & 3.59 & 0.14 & 0.14 & 0.08 & \\
\hline & & & Euro 2 & $\mathrm{~g} \cdot \mathrm{km}^{-1}$ & - & 0.93 & 10.28 & 2.37 & 0.14 & 0.14 & 0.07 & \\
\hline & & & Euro 3 & $\mathrm{~g} \cdot \mathrm{km}^{-1}$ & - & 0.52 & 7.11 & 0.85 & 0.03 & 0.03 & 0.04 & \\
\hline & & & Euro 4 & $\mathrm{~g} \cdot \mathrm{km}^{-1}$ & - & 0.20 & 2.36 & 0.28 & 0.01 & 0.01 & 0.03 & \\
\hline & & & Euro 5 & $\mathrm{~g} \cdot \mathrm{km}^{-1}$ & - & 0.16 & 2.01 & 0.26 & 0.01 & 0.01 & 0.02 & \\
\hline & & \multirow[t]{6}{*}{ Light-duty diesel vehicles } & Euro 0 & $\mathrm{~g} \cdot \mathrm{km}^{-1}$ & - & 1.99 & 10.05 & 2.36 & 1.19 & 1.07 & - & \multirow{6}{*}{$\begin{array}{l}\text { Estimated based on the } \\
\text { International Vehicle Emission } \\
\text { (IVE) Model (Huang et al., 2015) }\end{array}$} \\
\hline & & & Euro 1 & $\mathrm{~g} \cdot \mathrm{km}^{-1}$ & - & 1.46 & 2.70 & 0.43 & 0.42 & 0.38 & - & \\
\hline & & & Euro 2 & $\mathrm{~g} \cdot \mathrm{km}^{-1}$ & - & 1.46 & 3.38 & 0.28 & 0.35 & 0.31 & - & \\
\hline & & & Euro 3 & $\mathrm{~g} \cdot \mathrm{km}^{-1}$ & - & 1.26 & 1.05 & 0.14 & 0.22 & 0.19 & - & \\
\hline & & & Euro 4 & $\mathrm{~g} \cdot \mathrm{km}^{-1}$ & - & 1.02 & 0.98 & 0.10 & 0.20 & 0.19 & - & \\
\hline & & & Euro 5 & $\mathrm{~g} \cdot \mathrm{km}^{-1}$ & - & 1.02 & 0.98 & 0.10 & 0.20 & 0.19 & - & \\
\hline
\end{tabular}




\begin{tabular}{|c|c|c|c|c|c|c|c|c|c|c|c|c|}
\hline \multicolumn{4}{|c|}{ Source classification } & \multirow{2}{*}{ Units } & \multicolumn{7}{|c|}{ Emission factors } & \multirow{2}{*}{ References } \\
\hline 1st level & 2nd level & 3 rd level & & & $\mathrm{SO}_{2}$ & $\mathrm{NO}_{x}$ & $\mathrm{CO}$ & NMVOCs & $\mathrm{PM}_{10}$ & $\mathrm{PM}_{2,5}$ & $\mathrm{NH}_{3}$ & \\
\hline & & \multirow[t]{6}{*}{ Heavy-duty gasoline vehicles } & Euro 0 & $\mathrm{~g} \cdot \mathrm{km}^{-1}$ & - & 5.16 & 100.74 & 16.29 & 0.65 & 0.59 & 0.00 & \multirow{6}{*}{$\begin{array}{l}\text { Estimated based on the } \\
\text { International Vehicle Emission } \\
\text { (IVE) Model (Huang et al., 2015) }\end{array}$} \\
\hline & & & Euro 1 & $\mathrm{~g} \cdot \mathrm{km}^{-1}$ & - & 2.65 & 62.09 & 11.37 & 0.35 & 0.32 & 0.08 & \\
\hline & & & Euro 2 & $\mathrm{~g} \cdot \mathrm{km}^{-1}$ & - & 2.56 & 33.28 & 6.80 & 0.16 & 0.14 & 0.07 & \\
\hline & & & Euro 3 & $\mathrm{~g} \cdot \mathrm{km}^{-1}$ & - & 1.52 & 16.50 & 4.34 & 0.10 & 0.09 & 0.04 & \\
\hline & & & Euro 4 & $\mathrm{~g} \cdot \mathrm{km}^{-1}$ & - & 0.78 & 7.54 & 2.53 & 0.10 & 0.09 & 0.03 & \\
\hline & & & Euro 5 & $\mathrm{~g} \cdot \mathrm{km}^{-1}$ & - & 0.58 & 7.54 & 2.53 & 0.10 & 0.09 & 0.02 & \\
\hline & & \multirow[t]{6}{*}{ Heavy-duty diesel vehicles } & Euro 0 & $\mathrm{~g} \cdot \mathrm{km}^{-1}$ & - & 12.42 & 26.33 & 5.34 & 3.22 & 2.89 & - & \multirow{6}{*}{$\begin{array}{l}\text { Estimated based on the } \\
\text { International Vehicle Emission } \\
\text { (IVE) Model (Huang et al., 2015) }\end{array}$} \\
\hline & & & Euro 1 & $\mathrm{~g} \cdot \mathrm{km}^{-1}$ & - & 11.16 & 24.65 & 4.61 & 2.46 & 2.21 & - & \\
\hline & & & Euro 2 & $\mathrm{~g} \cdot \mathrm{km}^{-1}$ & - & 9.89 & 21.70 & 2.81 & 2.21 & 1.98 & - & \\
\hline & & & Euro 3 & $\mathrm{~g} \cdot \mathrm{km}^{-1}$ & - & 9.89 & 16.85 & 1.13 & 0.99 & 0.89 & - & \\
\hline & & & Euro 4 & $\mathrm{~g} \cdot \mathrm{km}^{-1}$ & - & 9.89 & 8.13 & 0.86 & 0.63 & 0.57 & - & \\
\hline & & & Euro 5 & $\mathrm{~g} \cdot \mathrm{km}^{-1}$ & - & 8.64 & 4.05 & 0.86 & 0.32 & 0.28 & - & \\
\hline & & \multirow[t]{6}{*}{ Heavy-duty gasoline truck } & Euro 0 & $\mathrm{~g} \cdot \mathrm{km}^{-1}$ & - & 7.26 & 153.91 & 18.07 & 0.82 & 0.73 & 0.00 & \multirow{6}{*}{$\begin{array}{l}\text { Estimated based on the } \\
\text { International Vehicle Emission } \\
\text { (IVE) Model (Huang et al., 2015) }\end{array}$} \\
\hline & & & Euro 1 & $\mathrm{~g} \cdot \mathrm{km}^{-1}$ & - & 3.72 & 94.74 & 17.80 & 0.44 & 0.40 & 0.08 & \\
\hline & & & Euro 2 & $\mathrm{~g} \cdot \mathrm{km}^{-1}$ & - & 3.63 & 36.44 & 8.42 & 0.20 & 0.18 & 0.07 & \\
\hline & & & Euro 3 & $\mathrm{~g} \cdot \mathrm{km}^{-1}$ & - & 2.14 & 16.73 & 4.29 & 0.12 & 0.11 & 0.04 & \\
\hline & & & Euro 4 & $\mathrm{~g} \cdot \mathrm{km}^{-1}$ & - & 1.13 & 7.03 & 2.29 & 0.12 & 0.11 & 0.03 & \\
\hline & & & Euro 5 & $\mathrm{~g} \cdot \mathrm{km}^{-1}$ & - & 0.85 & 7.03 & 2.29 & 0.12 & 0.11 & 0.02 & \\
\hline & & \multirow[t]{5}{*}{ Heavy-duty diesel truck } & Euro 0 & $\mathrm{~g} \cdot \mathrm{km}^{-1}$ & - & 16.75 & 10.54 & 8.46 & 2.24 & 2.04 & - & \multirow{5}{*}{$\begin{array}{l}\text { Estimated based on the } \\
\text { International Vehicle Emission } \\
\text { (IVE) Model (Huang et al., 2015) }\end{array}$} \\
\hline & & & Euro 1 & $\mathrm{~g} \cdot \mathrm{km}^{-1}$ & - & 11.62 & 6.73 & 3.72 & 1.60 & 1.44 & - & \\
\hline & & & Euro 2 & $\mathrm{~g} \cdot \mathrm{km}^{-1}$ & - & 9.61 & 3.58 & 2.16 & 1.29 & 1.16 & - & \\
\hline & & & Euro 3 & $\mathrm{~g} \cdot \mathrm{km}^{-1}$ & - & 9.61 & 3.24 & 1.06 & 0.88 & 0.79 & - & \\
\hline & & & Euro 4 & $\mathrm{~g} \cdot \mathrm{km}^{-1}$ & - & 8.41 & 2.56 & 0.67 & 0.47 & 0.43 & - & \\
\hline
\end{tabular}




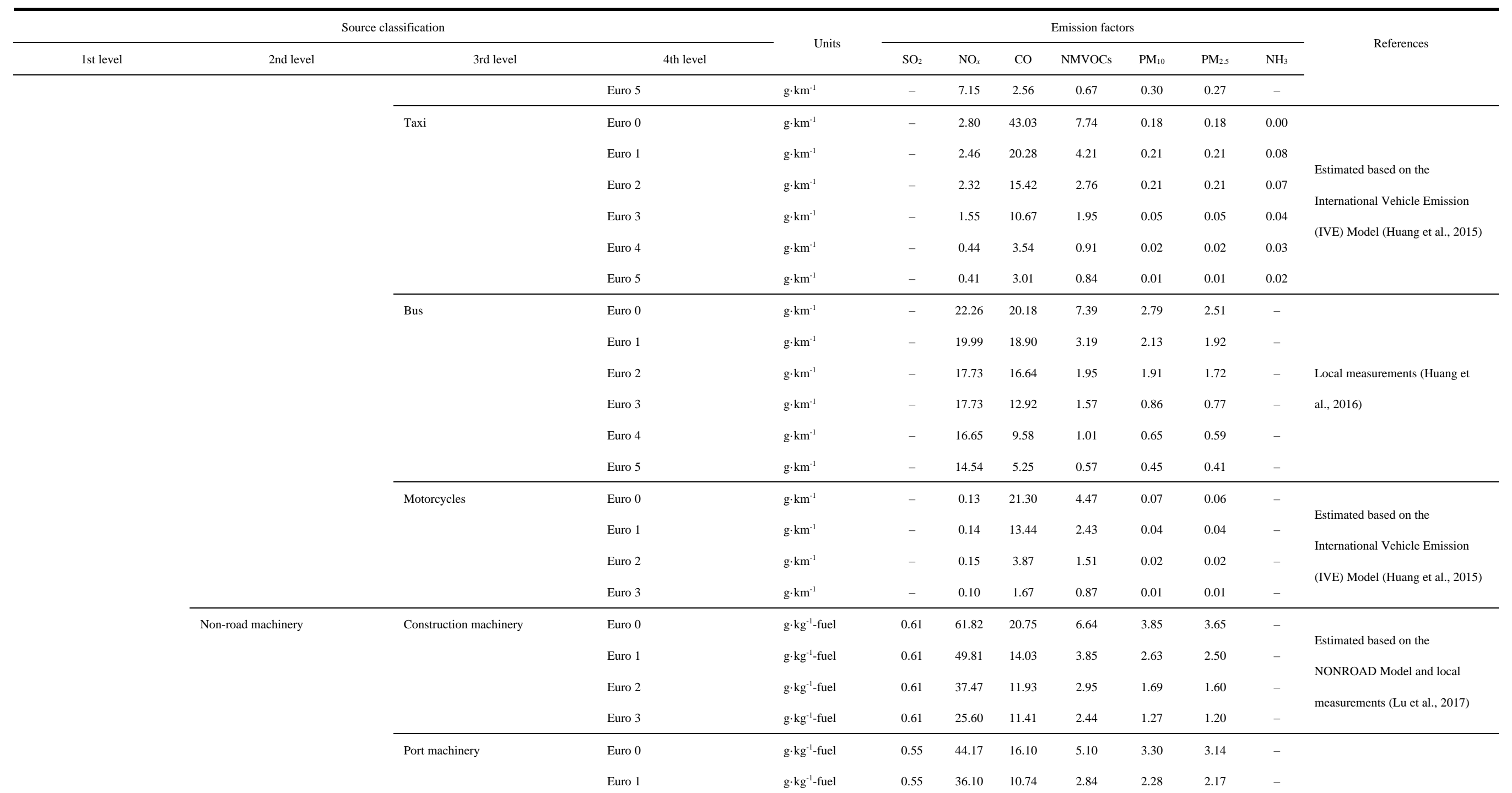




\begin{tabular}{|c|c|c|c|c|c|c|c|c|c|c|c|c|}
\hline \multicolumn{4}{|c|}{ Source classification } & \multirow{2}{*}{ Units } & \multicolumn{7}{|c|}{ Emission factors } & \multirow{2}{*}{ References } \\
\hline 1 st level & 2nd level & 3rd level & 4th level & & $\mathrm{SO}_{2}$ & $\mathrm{NO}_{x}$ & $\mathrm{CO}$ & NMVOCs & $\mathrm{PM}_{10}$ & $\mathrm{PM}_{2.5}$ & $\mathrm{NH}_{3}$ & \\
\hline & & & Euro 2 & $\mathrm{~g} \cdot \mathrm{kg}^{-1}$-fuel & 0.55 & 27.42 & 9.47 & 2.47 & 1.36 & 1.29 & - & \multirow{2}{*}{$\begin{array}{l}\text { Estimated based on the } \\
\text { NONROAD Model and local } \\
\text { measurements (Lu et al., 2017) }\end{array}$} \\
\hline & & & Euro 3 & $\mathrm{~g} \cdot \mathrm{kg}^{-1}$-fuel & 0.55 & 17.68 & 8.95 & 1.88 & 1.02 & 0.97 & - & \\
\hline & & \multirow[t]{4}{*}{ Factory machinery } & Euro 0 & $\mathrm{~g} \cdot \mathrm{kg}^{-1}$-fuel & 0.45 & 61.82 & 20.75 & 6.64 & 3.85 & 3.65 & - & \multirow{4}{*}{$\begin{array}{l}\text { Estimated based on the } \\
\text { NONROAD Model and local } \\
\text { measurements (Lu et al., } 2017\end{array}$} \\
\hline & & & Euro 1 & $\mathrm{~g} \cdot \mathrm{kg}^{-1}$-fuel & 0.45 & 49.81 & 14.03 & 3.85 & 2.63 & 2.50 & - & \\
\hline & & & Euro 2 & $\mathrm{~g} \cdot \mathrm{kg}^{-1}$-fuel & 0.45 & 37.47 & 11.93 & 2.95 & 1.69 & 1.60 & - & \\
\hline & & & Euro 3 & $\mathrm{~g} \cdot \mathrm{kg}^{-1}$-fuel & 0.45 & 25.60 & 11.41 & 2.44 & 1.27 & 1.20 & - & \\
\hline & & \multirow[t]{4}{*}{ Agricultural machinery } & Euro 0 & $\mathrm{~g} \cdot \mathrm{kg}^{-1}$-fuel & 0.52 & 56.63 & 39.43 & 11.86 & 5.12 & 4.86 & - & \multirow{4}{*}{$\begin{array}{l}\text { Estimated based on the } \\
\text { NONROAD Model and local } \\
\text { measurements (Lu et al., } 2017\end{array}$} \\
\hline & & & Euro 1 & $\mathrm{~g} \cdot \mathrm{kg}^{-1}$-fuel & 0.52 & 45.46 & 25.81 & 6.47 & 3.49 & 3.32 & - & \\
\hline & & & Euro 2 & $\mathrm{~g} \cdot \mathrm{kg}^{-1}$-fuel & 0.52 & 35.89 & 21.07 & 4.85 & 2.50 & 2.38 & - & \\
\hline & & & Euro 3 & $\mathrm{~g} \cdot \mathrm{kg}^{-1}$-fuel & 0.52 & 25.35 & 17.10 & 3.23 & 2.21 & 2.10 & - & \\
\hline & & \multirow[t]{4}{*}{ Garden equipment } & Euro 0 & $\mathrm{~g} \cdot \mathrm{kg}^{-1}$-fuel & - & 5.54 & 787.07 & 118.15 & 1.84 & 1.84 & - & \multirow{4}{*}{$\begin{array}{l}\text { Estimated based on the } \\
\text { NONROAD Model and local } \\
\text { measurements (Lu et al., 2017) }\end{array}$} \\
\hline & & & Euro 1 & $\mathrm{~g} \cdot \mathrm{kg}^{-1}$-fuel & - & 6.77 & 802.04 & 75.16 & 1.34 & 1.34 & - & \\
\hline & & & Euro 2 & $\mathrm{~g} \cdot \mathrm{kg}^{-1}$-fuel & - & 4.77 & 703.52 & 64.46 & 0.76 & 0.76 & - & \\
\hline & & & Euro 3 & $\mathrm{~g} \cdot \mathrm{kg}^{-1}$-fuel & - & 3.57 & 641.19 & 51.61 & 0.58 & 0.58 & - & \\
\hline & & \multirow[t]{4}{*}{ Ground handling equipment } & Euro 0 & $\mathrm{~g} \cdot \mathrm{kg}^{-1}$-fuel & 0.70 & 61.82 & 20.75 & 6.64 & 3.85 & 3.65 & - & \multirow{4}{*}{$\begin{array}{l}\text { Estimated based on the } \\
\text { NONROAD Model and local } \\
\text { measurements (Lu et al., 2017) }\end{array}$} \\
\hline & & & Euro 1 & $\mathrm{~g} \cdot \mathrm{kg}^{-1}$-fuel & 0.70 & 49.81 & 14.03 & 3.85 & 2.63 & 2.50 & - & \\
\hline & & & Euro 2 & $\mathrm{~g} \cdot \mathrm{kg}^{-1}$-fuel & 0.70 & 37.47 & 11.93 & 2.95 & 1.69 & 1.60 & - & \\
\hline & & & Euro 3 & $\mathrm{~g} \cdot \mathrm{kg}^{-1}$-fuel & 0.70 & 25.60 & 11.41 & 2.44 & 1.27 & 1.20 & - & \\
\hline & Marine & - & - & - & \multicolumn{8}{|c|}{ Detailed emission factors can be referred to Fan et al., 2016} \\
\hline & Aviation aircraft & - & - & - & \multicolumn{8}{|c|}{ Detailed emission factors can be referred to ICAO, 2014} \\
\hline \multirow[t]{2}{*}{ Dust sources } & Construction dust & - & - & $\mathrm{g} \cdot \mathrm{m}^{-2} \cdot \mathrm{month}^{-1}$ & - & - & - & - & 78.03 & 19.36 & - & MEP, 2014 \\
\hline & Road dust & highways & - & $\mathrm{g} \cdot \mathrm{km}^{-1}$ & - & - & - & - & 0.51 & 0.12 & - & MEP, 2014 \\
\hline
\end{tabular}




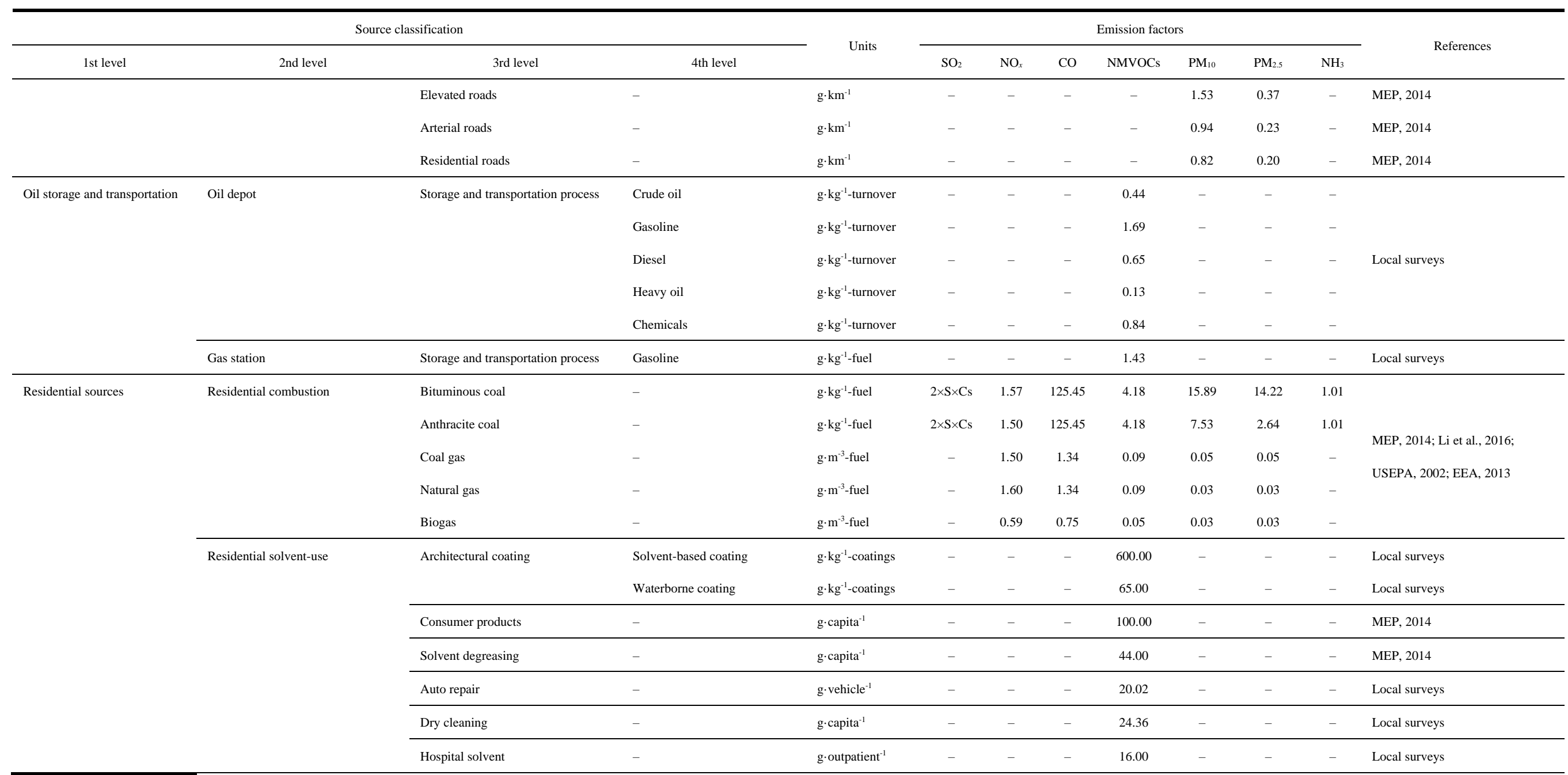




\begin{tabular}{|c|c|c|c|c|c|c|c|c|c|c|c|c|}
\hline \multicolumn{4}{|c|}{ Source classification } & \multirow{2}{*}{ Units } & \multicolumn{7}{|c|}{ Emission factors } & \multirow{2}{*}{ References } \\
\hline 1st level & 2nd level & 3rd level & 4th level & & $\mathrm{SO}_{2}$ & $\mathrm{NO}_{x}$ & $\mathrm{CO}$ & NMVOCs & $\mathrm{PM}_{10}$ & $\mathrm{PM}_{2.5}$ & $\mathrm{NH}_{3}$ & \\
\hline & Catering & - & - & $\mathrm{kg} \cdot$ restaurant ${ }^{-1}$ & - & - & - & 95.34 & 41.30 & 33.04 & - & $\begin{array}{l}\text { Local measurements (Wang et al., } \\
\text { 2018; Gao et al., 2019) }\end{array}$ \\
\hline & Human excretion & - & - & $\mathrm{kg} \cdot$ capita $^{-1}$ & - & - & - & - & - & - & 0.79 & MEP, 2014 \\
\hline \multirow[t]{3}{*}{ Waste treatment and disposal } & Sewage treatment & - & - & $\mathrm{g} \cdot \mathrm{t}^{-1}$-wastewater & - & - & - & 1.10 & - & - & 3.20 & MEP, 2014 \\
\hline & Waste treatment & Landfill & - & $\mathrm{g} \cdot \mathrm{kg}^{-1}$-refuse & - & - & - & 0.23 & - & - & 0.56 & MEP, 2014 \\
\hline & & Composting & - & $\mathrm{g} \cdot \mathrm{kg}^{-1}$-refuse & - & - & - & 0.74 & - & - & 1.28 & MEP, 2014 \\
\hline \multirow[t]{16}{*}{ Agricultural sources } & Livestock and poultry breedin & Swine breeding & Shed & $\mathrm{g} \cdot$ head $^{-1} \cdot \mathrm{d}^{-1}$ & - & - & - & - & - & - & 7.97 & \\
\hline & & & Manure fertilizing & g head ${ }^{-1} \cdot \mathrm{d}^{-1}$ & - & - & - & - & - & - & 1.80 & Local measurements (Chen, \\
\hline & & & Sewage storage & $\mathrm{g} \cdot$ head $^{-1} \cdot \mathrm{d}^{-1}$ & - & - & - & - & - & - & 1.68 & 2017a) \\
\hline & & & Apply fertilizer & $\mathrm{g} \cdot$ head $^{-1} \cdot \mathrm{d}^{-1}$ & - & - & - & - & - & - & 1.80 & \\
\hline & & Dairy cow breeding & Shed & $\mathrm{g} \cdot$ head $^{-1} \cdot \mathrm{d}^{-1}$ & - & - & - & - & - & - & 33.80 & \multirow{4}{*}{ Local measurements (Zhou, 2019) } \\
\hline & & & Manure fertilizing & $\mathrm{g} \cdot$ head $^{-1} \cdot \mathrm{d}^{-1}$ & - & - & - & - & - & - & 17.40 & \\
\hline & & & Sewage storage & $\mathrm{g} \cdot$ head $^{-1} \cdot \mathrm{d}^{-1}$ & - & - & - & - & - & - & 25.70 & \\
\hline & & & Apply fertilizer & $\mathrm{g} \cdot$ head $^{-1} \cdot \mathrm{d}^{-1}$ & - & - & - & - & - & - & 93.80 & \\
\hline & & Beef cattle & Shed & $\mathrm{g} \cdot$ head $^{-1} \cdot \mathrm{d}^{-1}$ & - & - & - & - & - & - & 43.00 & \multirow{4}{*}{ MEP, 2014} \\
\hline & & & Manure fertilizing & $\mathrm{g} \cdot$ head $^{-1} \cdot \mathrm{d}^{-1}$ & - & - & - & - & - & - & 20.00 & \\
\hline & & & Sewage storage & $\mathrm{g} \cdot$ head $^{-1} \cdot \mathrm{d}^{-1}$ & - & - & - & - & - & - & 20.00 & \\
\hline & & & Apply fertilizer & $\mathrm{g} \cdot$ head $^{-1} \cdot \mathrm{d}^{-1}$ & - & - & - & - & - & - & 22.50 & \\
\hline & & \multirow[t]{4}{*}{ Sheep } & Shed & $\mathrm{g} \cdot$ head $^{-1} \cdot \mathrm{d}^{-1}$ & - & - & - & - & - & - & 12.10 & \multirow{4}{*}{ MEP, 2014} \\
\hline & & & Manure fertilizing & $\mathrm{g} \cdot$ head $^{-1} \cdot \mathrm{d}^{-1}$ & - & - & - & - & - & - & 5.60 & \\
\hline & & & Sewage storage & $\mathrm{g} \cdot$ head $^{-1} \cdot \mathrm{d}^{-1}$ & - & - & - & - & - & - & 5.60 & \\
\hline & & & Apply fertilizer & $\mathrm{g} \cdot$ head $^{-1} \cdot \mathrm{d}^{-1}$ & - & - & - & - & - & - & 6.30 & \\
\hline
\end{tabular}




\begin{tabular}{|c|c|c|c|c|c|c|c|c|c|c|c|c|}
\hline \multicolumn{4}{|c|}{ Source classification } & \multirow{2}{*}{ Units } & \multicolumn{7}{|c|}{ Emission factors } & \multirow{2}{*}{ References } \\
\hline 1st level & 2nd level & 3rd level & 4th level & & $\mathrm{SO}_{2}$ & $\mathrm{NO}_{x}$ & $\mathrm{CO}$ & NMVOCs & $\mathrm{PM}_{10}$ & $\mathrm{PM}_{2.5}$ & $\mathrm{NH}_{3}$ & \\
\hline & & Broiler & Shed & $\mathrm{g} \cdot$ head- $1 \cdot \mathrm{d}-1$ & - & - & - & - & - & - & 0.10 & \multirow{3}{*}{ MEP, 2014} \\
\hline & & & Manure fertilizing & $\mathrm{g} \cdot$ head- $1 \cdot \mathrm{d}-1$ & - & - & - & - & - & - & 0.10 & \\
\hline & & & Apply fertilizer & $\mathrm{g} \cdot$ head- $1 \cdot \mathrm{d}-1$ & - & - & - & - & - & - & 0.03 & \\
\hline & & \multirow[t]{2}{*}{ Egg-laying poultry } & Shed & $\mathrm{g} \cdot$ head $-1 \cdot \mathrm{d}-1$ & - & - & - & - & - & - & 1.40 & \multirow{2}{*}{ MEP, 2014} \\
\hline & & & Apply fertilizer & $g \cdot$ head- $1 \cdot d-1$ & - & - & - & - & - & - & 0.27 & \\
\hline & \multirow[t]{2}{*}{$\mathrm{N}$-fertilizer application } & Paddy field & - & $\mathrm{g} \cdot \mathrm{kg}^{-1}$-fertilizer & - & - & - & - & - & - & 243.40 & $\begin{array}{l}\text { Local measurements (Chen et al., } \\
\text { 2017b) }\end{array}$ \\
\hline & & Wheat field & - & $\mathrm{g} \cdot \mathrm{kg}^{-1}$-fertilizer & - & - & - & - & - & - & 74.70 & $\begin{array}{l}\text { Local measurements (Xia et al., } \\
\text { 2018) }\end{array}$ \\
\hline & \multirow[t]{10}{*}{ Biomass burning } & Field burning & Soybean & $\mathrm{g} \cdot \mathrm{kg}^{-1}$-straw & 0.53 & 1.08 & 32.30 & 8.45 & 1.98 & 1.84 & 0.53 & \multirow{4}{*}{$\begin{array}{l}\text { Local measurements (Tang et al., } \\
\text { 2014); MEP, } 2014\end{array}$} \\
\hline & & & Rice stalk & $\mathrm{g} \cdot \mathrm{kg}^{-1}$-straw & 0.53 & 1.42 & 27.70 & 8.45 & 3.26 & 3.03 & 0.53 & \\
\hline & & & Wheat stalk & $\mathrm{g} \cdot \mathrm{kg}^{-1}$-straw & 0.85 & 1.19 & 20.60 & 7.48 & 2.57 & 2.39 & 0.37 & \\
\hline & & & Corn stalk & $\mathrm{g} \cdot \mathrm{kg}^{-1}$-straw & 0.44 & 4.30 & 53.00 & 10.00 & 12.58 & 11.70 & 0.68 & \\
\hline & & \multirow[t]{6}{*}{ Household stove } & Soybean & $\mathrm{g} \cdot \mathrm{kg}^{-1}$-straw & 1.36 & 2.19 & 80.70 & 7.97 & 8.29 & 7.71 & 0.52 & \multirow{6}{*}{$\begin{array}{l}\text { Local measurements (Tang et al., } \\
\text { 2014); MEP, } 2014\end{array}$} \\
\hline & & & Rape & $\mathrm{g} \cdot \mathrm{kg}^{-1}$-straw & 1.36 & 1.65 & 133.50 & 7.97 & 13.73 & 12.77 & 0.52 & \\
\hline & & & Rice stalk & $\mathrm{g} \cdot \mathrm{kg}^{-1}$-straw & 0.48 & 2.02 & 82.00 & 7.36 & 9.83 & 9.14 & 0.52 & \\
\hline & & & Straw & $\mathrm{g} \cdot \mathrm{kg}^{-1}$-straw & 2.36 & 0.86 & 191.55 & 13.74 & 7.40 & 6.88 & 0.37 & \\
\hline & & & Corn stalk & $\mathrm{g} \cdot \mathrm{kg}^{-1}$-straw & 1.33 & 1.16 & 108.90 & 5.46 & 6.17 & 5.74 & 0.68 & \\
\hline & & & Firewood & $\mathrm{g} \cdot \mathrm{kg}^{-1}$-straw & 0.40 & 1.28 & 67.53 & 1.73 & 4.68 & 4.36 & 1.30 & \\
\hline
\end{tabular}


235 Table S2. Emission estimation methods and activity data sources.

\begin{tabular}{|c|c|c|c|c|c|}
\hline \multirow{2}{*}{ No. } & \multicolumn{2}{|c|}{ Sources } & \multirow{2}{*}{ Estimation methods } & \multirow{2}{*}{ Activity data source } & \multirow{2}{*}{$\begin{array}{c}\text { Reliability } \\
\text { level }\end{array}$} \\
\hline & 1st level & 2nd level & & & \\
\hline \multirow[t]{2}{*}{1} & Stationary combustion sources & - & $\mathrm{SO}_{2}, \mathrm{PM}_{10}$, and $\mathrm{PM}_{2.5}$ : mass balance method; & 2017 environmental statistics & A \\
\hline & & & Other pollutants: emission factor method & & \\
\hline 2 & Industrial process sources & - & Emission factor method & 2017 environmental statistics & $\mathrm{B}$ \\
\hline 3 & Industrial solvent-use sources & - & Mass balance method & 2017 environmental statistics & $\mathrm{C}$ \\
\hline \multirow[t]{4}{*}{4} & Mobile sources & Motor vehicles & International Vehicle Emission (IVE) model & Local surveys & $\mathrm{B}$ \\
\hline & & Non-road machinery & Emission factor method & Estimated based on local surveys & $\mathrm{D}$ \\
\hline & & Ships & Automatic Identification System (AIS) method & 2017 AIS data & $\mathrm{B}$ \\
\hline & & Aviation aircraft & Emission factor and FOA3.0 method (Wayson et al., 2009) & Local flight information surveys & $\mathrm{B}$ \\
\hline \multirow[t]{2}{*}{5} & Dust sources & Construction dust & Emission factor method & Estimated based on statistic yearbooks & $\mathrm{D}$ \\
\hline & & Road dust & Emission factor method & Local surveys & $\mathrm{B}$ \\
\hline 6 & Oil storage and transportation sources & - & Emission factor method & Statistic yearbooks & $\mathrm{C}$ \\
\hline \multirow[t]{4}{*}{7} & Residential sources & Residential combustion & Emission factor method & Statistic yearbooks & $\mathrm{C}$ \\
\hline & & Residential solvent-use & Emission factor method & Estimated based on statistic yearbooks & $\mathrm{D}$ \\
\hline & & Catering & Emission factor method & Estimated based on statistic yearbooks & $\mathrm{D}$ \\
\hline & & Human excretion & Emission factor method & Estimated based on statistic yearbooks & $\mathrm{D}$ \\
\hline 8 & Waste treatment and disposal sources & - & Emission factor method & Statistic yearbooks & $\mathrm{C}$ \\
\hline \multirow[t]{3}{*}{9} & Agricultural sources & Livestock and poultry breeding & Emission factor method & Statistic yearbooks & $\mathrm{C}$ \\
\hline & & $\mathrm{N}$-fertilizer application & Emission factor method & Statistic yearbooks & $\mathrm{C}$ \\
\hline & & Biomass burning & Emission factor method & Estimated based on statistic yearbooks & $\mathrm{D}$ \\
\hline
\end{tabular}


237 Table S3. Emission estimation methods and activity data sources.

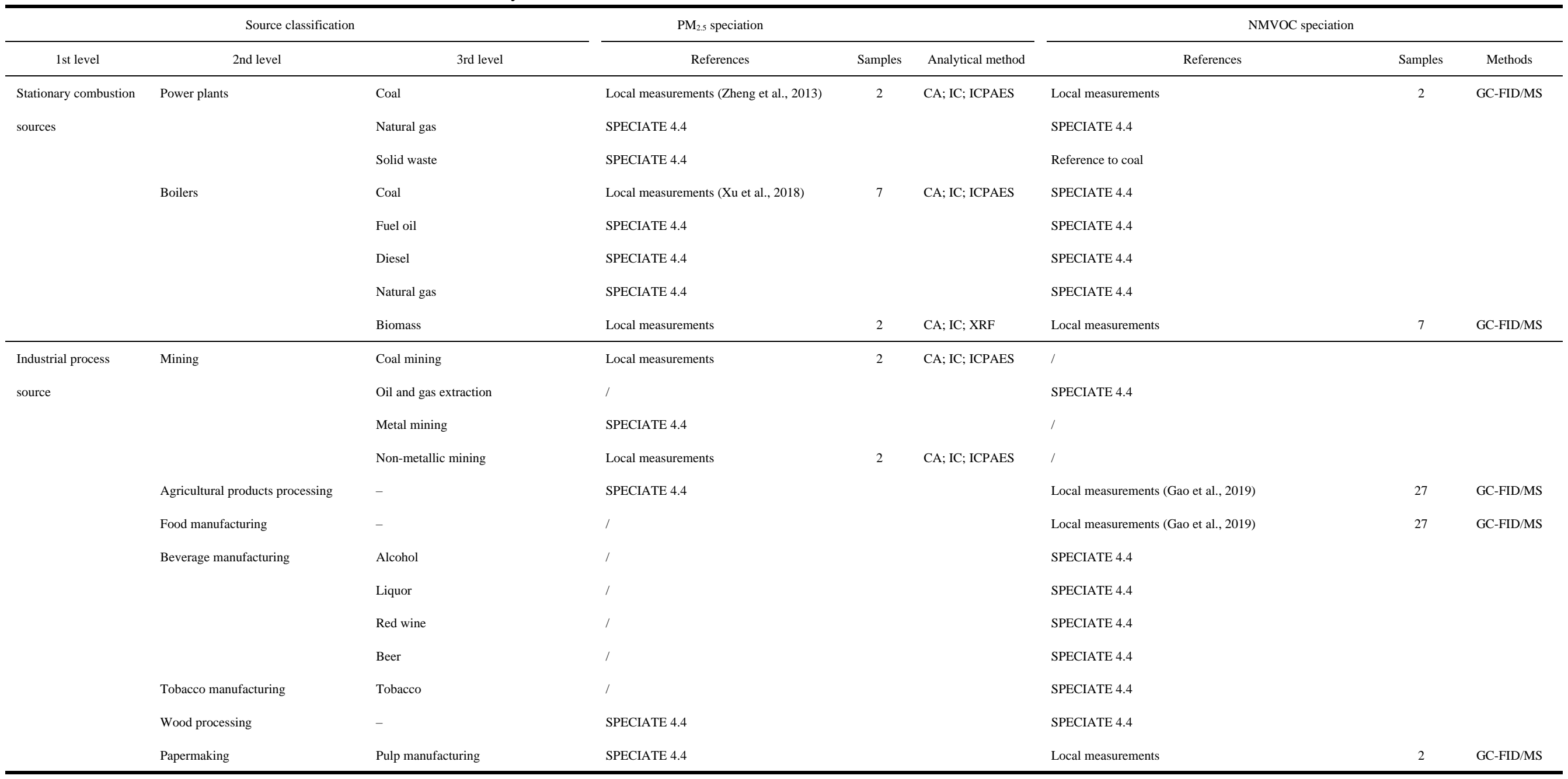




\begin{tabular}{|c|c|c|c|c|c|c|c|}
\hline \multirow[t]{7}{*}{ Petroleum refining } & Process devices & l & & & Local measurements (Wang et al., 2017a) & 21 & GC-FID/MS \\
\hline & Equipment leak & 1 & & & Local measurements (Wang et al., 2017a) & 21 & GC-FID/MS \\
\hline & Storage tanks & ' & & & Local measurements (Wang et al., 2017a) & 8 & GC-FID/MS \\
\hline & Loading and unloading & ' & & & Local measurements (Wang et al., 2017a) & 8 & GC-FID/MS \\
\hline & Flares & ' & & & SPECIATE 4.4 & & \\
\hline & Wastewater treatment & l & & & Local measurements (Wang et al., 2017a) & 6 & GC-FID/MS \\
\hline & Petrochemical furnace & l & & & SPECIATE 4.4 & & \\
\hline Coking & Coke production & Local measurements & 2 & CA; IC; ICPAES & Local measurements (Wang et al., 2017a; Jing et al., 2018) & 4 & GC-FID/MS \\
\hline \multirow[t]{11}{*}{ Chemical manufacturing } & Organic chemical manufacturing & l & & & Local measurements (Wang et al., 2017a; Jing et al., 2018) & 32 & GC-FID/MS \\
\hline & Inorganic chemical manufacturing & SPECIATE 4.4 & & & I & & \\
\hline & Fertilizer & SPECIATE 4.4 & & & I & & \\
\hline & Pesticide & ' & & & SPECIATE 4.4 & & \\
\hline & Ink production & ' & & & Local measurements (Wang et al., 2017a; Jing et al., 2018) & 22 & GC-FID/MS \\
\hline & Synthetic resin & I & & & Local measurements (Wang et al., 2017a; Jing et al., 2018) & 2 & GC-FID/MS \\
\hline & Synthetic rubber & ' & & & Local measurements (Wang et al., 2017a; Jing et al., 2018) & 6 & GC-FID/MS \\
\hline & Synthetic fiber monomer & ' & & & Local measurements (Wang et al., 2017a; Jing et al., 2018) & 2 & GC-FID/MS \\
\hline & Specialty chemicals & SPECIATE 4.4 & & & SPECIATE 4.4 & & \\
\hline & Household chemicals & I & & & SPECIATE 4.4 & & \\
\hline & Chemical fiber & ' & & & Local measurements (Wang et al., 2017a; Jing et al., 2018) & 2 & GC-FID/MS \\
\hline \multirow[t]{2}{*}{ Rubber and plastic manufacturing } & Rubber products & I & & & Local measurements (Wang et al., 2017a; Jing et al., 2018) & 6 & GC-FID/MS \\
\hline & Plastic products & I & & & Local measurements (Wang et al., 2017a; Jing et al., 2018) & 2 & GC-FID/MS \\
\hline \multirow[t]{2}{*}{ Non-metallic mineral manufacturing } & Cement manufacturing & SPECIATE 4.4 & & & I & & \\
\hline & Lime manufacturing & SPECIATE 4.4 & & & I & & \\
\hline
\end{tabular}




\begin{tabular}{|c|c|c|c|c|c|}
\hline & Plaster manufacturing & SPECIATE 4.4 & & & 1 \\
\hline & Gypsum board & SPECIATE 4.4 & & & । \\
\hline & Commodity concrete & Local measurements & 2 & CA; IC; ICPAES & / \\
\hline & Crushed Stone processing & SPECIATE 4.4 & & & I \\
\hline & Abrasive processing & SPECIATE 4.4 & & & । \\
\hline & Asphalt products & SPECIATE 4.4 & & & SPECIATE 4.4 \\
\hline & Glass products & SPECIATE 4.4 & & & । \\
\hline & Glass fiber & SPECIATE 4.4 & & & I \\
\hline & Ceramic products & SPECIATE 4.4 & & & SPECIATE 4.4 \\
\hline & Refractory products & SPECIATE 4.4 & & & । \\
\hline Ferrous metal manufacturing & Raw material yard & Local measurements & 2 & CA; IC; XRF & । \\
\hline & Sintering & Local measurements & 4 & CA; IC; XRF & SPECIATE 4.4 \\
\hline & Blast furnace & Local measurements & 3 & CA; IC; XRF & SPECIATE 4.4 \\
\hline & Converter & Local measurements & 2 & $\mathrm{CA} ; \mathrm{IC} ; \mathrm{XRF}$ & SPECIATE 4.4 \\
\hline & Electric furnace & SPECIATE 4.4 & & & SPECIATE 4.4 \\
\hline & Casing steel & SPECIATE 4.4 & & & SPECIATE 4.4 \\
\hline & Rolling steel & SPECIATE 4.4 & & & SPECIATE 4.4 \\
\hline & Ferroalloy production & SPECIATE 4.4 & & & I \\
\hline Non-ferrous metal manufacturing & Primary copper & SPECIATE 4.4 & & & । \\
\hline & Primary aluminum & SPECIATE 4.4 & & & I \\
\hline & Primary lead & SPECIATE 4.4 & & & । \\
\hline & Primary zinc & SPECIATE 4.4 & & & I \\
\hline & Secondary copper & SPECIATE 4.4 & & & 1 \\
\hline
\end{tabular}




\begin{tabular}{|c|c|c|c|c|c|c|c|c|}
\hline & \multirow{4}{*}{ Other miscellaneous sources } & \multirow{2}{*}{$\begin{array}{l}\text { Secondary aluminum } \\
\text { Secondary lead }\end{array}$} & \multicolumn{3}{|l|}{ SPECIATE 4.4} & \multicolumn{3}{|l|}{ SPECIATE 4.4} \\
\hline & & & SPECIATE 4.4 & & & \multicolumn{3}{|l|}{1} \\
\hline & & Secondary zinc & SPECIATE 4.4 & & & \multicolumn{3}{|l|}{ । } \\
\hline & & Electric arc welding & SPECIATE 4.4 & & & \multicolumn{3}{|l|}{ । } \\
\hline Industrial solvent-use & Textile & - & & & & Local measurements (Wang et al., 2017a; Jing et al., 2018) & 14 & GC-FID/MS \\
\hline \multirow[t]{11}{*}{ source } & Leather tanning & - & & & & SPECIATE 4.4 & & \\
\hline & Furniture manufacturing & - & & & & Local measurements (Wang et al., 2017a; Jing et al., 2018) & 6 & GC-FID/MS \\
\hline & Package and printing & - & & & & Local measurements (Wang et al., 2017a; Jing et al., 2018) & 8 & GC-FID/MS \\
\hline & Pharmaceutical manufacturing & - & & & & \multicolumn{3}{|l|}{ SPECIATE 4.4} \\
\hline & Shoemaking & - & & & & Local measurements (Wang et al., 2017a; Jing et al., 2018) & 6 & GC-FID/MS \\
\hline & Metal products & - & & & & Local measurements (Wang et al., 2017a; Jing et al., 2018) & 12 & GC-FID/MS \\
\hline & Machinery manufacturing & - & & & & \multicolumn{3}{|l|}{ Reference to Metal products } \\
\hline & Auto manufacturing & - & & & & Local measurements (Wang et al., 2017a; Jing et al., 2018) & 25 & GC-FID/MS \\
\hline & Shipbuilding & - & & & & Local measurements (Wang et al., 2017a; Jing et al., 2018) & 28 & GC-FID/MS \\
\hline & Appliance manufacturing & - & & & & \multicolumn{3}{|l|}{ SPECIATE 4.4} \\
\hline & Electronic equipment & - & & & & \multicolumn{3}{|l|}{ SPECIATE 4.4} \\
\hline \multirow[t]{7}{*}{ Mobile source } & Motor vehicles & Light-duty gasoline vehicles (LDGV) & Local measurements & 10 & CA; IC; XRF & Local measurements (Wang et al., 2017b) & 21 & GC-FID/MS \\
\hline & & Light-duty diesel vehicles (LDDV) & Reference to HDDT & & & \multicolumn{3}{|l|}{ Reference to HDDT } \\
\hline & & Heavy-duty gasoline vehicles (HDGV) & Reference to LDGV & & & \multicolumn{3}{|l|}{ Reference to LDGV } \\
\hline & & Heavy-duty diesel vehicles (HDDV) & Reference to HDDT & & & \multicolumn{3}{|l|}{ Reference to HDDT } \\
\hline & & Heavy-duty gasoline truck (HDGT) & Reference to LDGV & & & \multicolumn{3}{|l|}{ Reference to LDGV } \\
\hline & & Heavy-duty diesel truck (HDDT) & Local measurements & 15 & CA; IC; XRF & Local measurements (Wang et al., 2017b) & 16 & GC-FID/MS \\
\hline & & Taxi & Reference to LDGV & & & \multicolumn{3}{|l|}{ Reference to LDGV } \\
\hline
\end{tabular}




\begin{tabular}{|c|c|c|c|c|c|c|c|c|}
\hline & \multirow{7}{*}{ Non-road machinery } & Bus & \multicolumn{3}{|l|}{ Reference to HDDT } & Local measurements (Wang et al., 2017b) & 6 & GC-FID/MS \\
\hline & & Motorcycles & \multicolumn{3}{|l|}{ Reference to LDGV } & Local measurements (Wang et al., 2017b) & 7 & GC-FID/MS \\
\hline & & Evaporative emission & \multicolumn{3}{|l|}{ । } & \multicolumn{3}{|l|}{ Reference to Gas station } \\
\hline & & Port machinery & Local measurements & 2 & $\mathrm{CA} ; \mathrm{IC} ; \mathrm{XRF}$ & Local measurements & 2 & GC-FID/MS \\
\hline & & Factory machinery & Local measurements & 2 & $\mathrm{CA}$; IC; XRF & Local measurements & 2 & GC-FID/MS \\
\hline & & Agricultural machinery & Local measurements & 2 & CA; IC; XRF & Local measurements & 2 & GC-FID/MS \\
\hline & & \multicolumn{4}{|l|}{ Garden equipment } & \multicolumn{3}{|l|}{ Reference to LDGV } \\
\hline & & Ground handling equipment (GHE) & Local measurements & 2 & $\mathrm{CA}$; IC; XRF & Local measurements & 2 & GC-FID/MS \\
\hline & Marine & Inland ship & Local measurements & 2 & CA; IC; XRF & Reference to non-road diesel machinery & & \\
\hline & & Marine vessel & Local measurements & 1 & CA; IC; XRF & Local measurements (Huang et al., 2018c) & 11 & GC-FID/MS \\
\hline & Aviation aircraft & - & SPECIATE 4.4 & & & SPECIATE 4.4 & & \\
\hline Dust source & Construction dust & - & Local measurements & 3 & CA; IC; ICPAES & I & & \\
\hline \multirow{6}{*}{$\begin{array}{l}\text { Oil storage and } \\
\text { transportation source }\end{array}$} & Oil depot & Crude oil & I & & & Local measurements (Wang et al., 2017a) & 4 & GC-FID/MS \\
\hline & & Gasoline & 1 & & & Local measurements (Wang et al., 2017a) & 2 & GC-FID/MS \\
\hline & & Diesel & I & & & Local measurements (Wang et al., 2017a) & 2 & GC-FID/MS \\
\hline & & Heavy oil & l & & & Reference to Crude oil & & \\
\hline & & Chemicals & ' & & & SPECIATE 4.4 & & \\
\hline & Gas station & Gasoline & I & & & Local measurements (Wang et al., 2017a) & 4 & GC-FID/MS \\
\hline \multirow[t]{5}{*}{ Residential source } & Residential combustion & - & Local measurements & 2 & CA; IC; ICPAES & SPECIATE 4.4 & & \\
\hline & Residential solvent-use & Architectural coating & । & & & Local measurements (Wang et al., 2014b; Wang et al., 2016) & 4 & GC-FID/MS \\
\hline & & Consumer products & l & & & SPECIATE 4.4 & & \\
\hline & & Solvent degreasing & I & & & SPECIATE 4.4 & & \\
\hline & & Auto repair & I & & & Local measurements & 4 & GC-FID/MS \\
\hline
\end{tabular}




\begin{tabular}{|c|c|c|c|c|c|c|c|c|}
\hline & & Dry cleaning & l & & & SPECIATE 4.4 & & \\
\hline & & Hospital solvent & 1 & & & Local measurements & & \\
\hline & Catering & - & Local measurements & 27 & $\mathrm{CA} ; \mathrm{IC} ; \mathrm{XRF}$ & Local measurements (Gao et al., 2019) & 27 & GC-FID/MS \\
\hline \multirow{3}{*}{$\begin{array}{l}\text { Waste treatment and } \\
\text { disposal source }\end{array}$} & Sewage treatment & - & l & & & SPECIATE 4.4 & & \\
\hline & Waste treatment & Landfill & l & & & SPECIATE 4.4 & & \\
\hline & & Composting & l & & & SPECIATE 4.4 & & \\
\hline \multirow[t]{10}{*}{ Agricultural source } & Biomass burning & Field burning-Soybean & Local measurements (Tang et al., 2014) & 1 & CA; IC; ICPAES & Local measurements (Wang et al., 2014a) & 1 & GC-FID/MS \\
\hline & & Field burning-Rape & Local measurements (Tang et al., 2014) & 2 & CA; IC; ICPAES & Local measurements (Wang et al., 2014a) & 2 & GC-FID/MS \\
\hline & & Field burning-Rice stalk & Local measurements (Tang et al., 2014) & 2 & CA; IC; ICPAES & Local measurements (Wang et al., 2014a) & 2 & GC-FID/MS \\
\hline & & Field burning-Wheat stalk & Local measurements (Tang et al., 2014) & 2 & CA; IC; ICPAES & Local measurements (Wang et al., 2014a) & 2 & GC-FID/MS \\
\hline & & Field burning-Corn stalk & Local measurements (Tang et al., 2014) & 2 & CA; IC; ICPAES & Local measurements (Wang et al., 2014a) & 2 & GC-FID/MS \\
\hline & & Household stove-Soybean & Local measurements (Tang et al., 2014) & 2 & CA; IC; ICPAES & Local measurements (Wang et al., 2014a) & 2 & GC-FID/MS \\
\hline & & Household stove-Rape & Local measurements (Tang et al., 2014) & 2 & CA; IC; ICPAES & Local measurements (Wang et al., 2014a) & 2 & GC-FID/MS \\
\hline & & Household stove-Straw & Local measurements (Tang et al., 2014) & 2 & CA; IC; ICPAES & Local measurements (Wang et al., 2014a) & 2 & GC-FID/MS \\
\hline & & Household stove-Corn stalk & Local measurements (Tang et al., 2014) & 2 & CA; IC; ICPAES & Local measurements (Wang et al., 2014a) & 2 & GC-FID/MS \\
\hline & & Household stove-Firewood & Local measurements (Tang et al., 2014) & 1 & CA; IC; ICPAES & Local measurements (Wang et al., 2014a) & 1 & GC-FID/MS \\
\hline
\end{tabular}


Table S4. Annual air pollutant emissions in each city of the YRD region in 2017.

\begin{tabular}{|c|c|c|c|c|c|c|c|c|}
\hline \multirow{2}{*}{ Province } & \multirow{2}{*}{ City } & \multicolumn{7}{|c|}{ Annual air pollutant emissions (Gg/year) } \\
\hline & & $\mathrm{SO}_{2}$ & $\mathrm{NO}_{x}$ & $\mathrm{CO}$ & NMVOCs & $\mathrm{PM}_{10}$ & $\mathrm{PM}_{2.5}$ & $\mathrm{NH}_{3}$ \\
\hline Shanghai & Shanghai & 57.2 & 224.7 & 1392.9 & 418.2 & 123.7 & 56.2 & 54.4 \\
\hline \multirow{13}{*}{ Jiangsu } & Nanjing & 51.7 & 131.2 & 1445.6 & 223.3 & 90.8 & 39.7 & 29.1 \\
\hline & Wuxi & 42.0 & 94.6 & 1141.4 & 198.4 & 92.0 & 43.2 & 27.2 \\
\hline & Xuzhou & 94.7 & 148.5 & 1683.2 & 148.8 & 207.0 & 94.1 & 164.3 \\
\hline & Changzhou & 58.0 & 86.8 & 1313.7 & 153.1 & 118.9 & 44.8 & 26.7 \\
\hline & Suzhou & 105.4 & 183.4 & 2257.3 & 263.5 & 149.7 & 77.3 & 34.5 \\
\hline & Nantong & 40.4 & 81.2 & 2308.1 & 209.1 & 106.7 & 40.4 & 145.7 \\
\hline & Lianyungang & 25.2 & 72.2 & 826.3 & 117.6 & 87.6 & 33.6 & 96.4 \\
\hline & Huaian & 43.0 & 76.5 & 1978.3 & 105.9 & 109.7 & 34.5 & 104.1 \\
\hline & Yancheng & 42.5 & 77.3 & 1094.7 & 132.6 & 127.8 & 47.6 & 214.3 \\
\hline & Yangzhou & 24.8 & 52.1 & 930.1 & 139.5 & 104.8 & 34.2 & 51.6 \\
\hline & Zhenjiang & 42.7 & 55.2 & 1004.8 & 119.8 & 71.5 & 30.1 & 23.0 \\
\hline & Taizhou & 22.0 & 55.0 & 835.4 & 152.8 & 114.2 & 37.3 & 68.8 \\
\hline & Suqian & 26.6 & 51.4 & 490.4 & 91.6 & 59.5 & 20.5 & 107.1 \\
\hline \multirow{11}{*}{ Zhejiang } & Hangzhou & 39.0 & 110.7 & 1204.2 & 177.7 & 99.4 & 39.5 & 56.1 \\
\hline & Ningbo & 61.5 & 136.9 & 1546.4 & 347.9 & 124.8 & 57.7 & 35.5 \\
\hline & Wenzhou & 14.8 & 62.9 & 465.1 & 124.0 & 62.4 & 20.0 & 38.6 \\
\hline & Jiaxing & 33.4 & 68.9 & 1055.0 & 190.0 & 84.1 & 30.8 & 26.1 \\
\hline & Huzhou & 52.5 & 46.1 & 466.7 & 120.6 & 69.0 & 31.2 & 27.0 \\
\hline & Shaoxing & 27.1 & 56.8 & 646.9 & 148.3 & 67.4 & 24.6 & 42.1 \\
\hline & Jinhua & 27.8 & 59.2 & 498.7 & 100.6 & 70.5 & 28.5 & 38.9 \\
\hline & Quzhou & 41.0 & 46.2 & 534.5 & 60.4 & 50.5 & 24.8 & 42.1 \\
\hline & Zhoushan & 5.5 & 15.8 & 97.6 & 47.6 & 21.8 & 8.6 & 3.1 \\
\hline & Taizhou & 22.2 & 55.2 & 412.0 & 129.5 & 84.9 & 29.6 & 31.6 \\
\hline & Lishui & 13.8 & 17.9 & 109.0 & 36.9 & 40.5 & 12.3 & 21.6 \\
\hline \multirow{11}{*}{ Anhui } & Hefei & 21.5 & 98.7 & 907.1 & 149.1 & 135.0 & 55.1 & 73.1 \\
\hline & Wuhu & 39.4 & 85.2 & 815.3 & 82.9 & 99.0 & 43.5 & 34.3 \\
\hline & Bengbu & 15.8 & 40.3 & 599.4 & 63.5 & 116.1 & 40.7 & 79.2 \\
\hline & Huainan & 19.6 & 48.6 & 848.3 & 27.2 & 74.3 & 41.5 & 61.9 \\
\hline & Ma'anshan & 26.5 & 42.9 & 599.5 & 28.8 & 75.8 & 36.7 & 15.7 \\
\hline & Huaibei & 36.9 & 45.2 & 4437.3 & 37.5 & 74.3 & 44.1 & 21.0 \\
\hline & Tongling & 44.3 & 58.3 & 598.8 & 26.2 & 72.1 & 40.8 & 14.9 \\
\hline & Anqing & 41.7 & 48.0 & 671.1 & 78.5 & 86.9 & 37.0 & 62.4 \\
\hline & Huangshan & 6.5 & 8.7 & 134.8 & 17.1 & 22.8 & 7.0 & 21.5 \\
\hline & Chuzhou & 19.3 & 58.8 & 590.3 & 81.1 & 107.2 & 46.4 & 90.5 \\
\hline & Fuyang & 33.8 & 78.1 & 524.2 & 77.0 & 133.0 & 63.1 & 145.7 \\
\hline
\end{tabular}




\begin{tabular}{llllllll} 
Suzhou & 35.2 & 75.1 & 594.9 & 71.5 & 106.2 & 53.4 & 132.3 \\
Lu'an & 18.0 & 54.0 & 323.2 & 51.2 & 81.9 & 35.5 & 73.8 \\
Bozhou & 16.4 & 48.6 & 318.8 & 49.6 & 75.6 & 35.5 & 80.5 \\
Chizhou & 19.9 & 32.0 & 338.8 & 22.4 & 73.3 & 28.0 & 17.5 \\
Xuancheng & 27.4 & 46.7 & 446.1 & 45.9 & 81.8 & 33.3 & 32.9 \\
\hline
\end{tabular}


Table S5. Annual air pollutant emissions from different source in the YRD region in 2017.

\begin{tabular}{|c|c|c|c|c|c|c|c|c|}
\hline $1^{\text {st }}$ level & $2^{\text {nd }}$ level & $\mathrm{SO}_{2}$ & $\mathrm{NO}_{x}$ & $\mathrm{CO}$ & NMVOCs & $\mathrm{PM}_{10}$ & $\mathrm{PM}_{2.5}$ & $\mathrm{NH}_{3}$ \\
\hline Power plants & Power plants & 247.3 & 446.5 & 1444.0 & 12.1 & 156.0 & 105.5 & 11.4 \\
\hline Boilers & Boilers & 756.1 & 418.0 & 28259.7 & 9.5 & 62.5 & 36.6 & 1.5 \\
\hline \multirow{13}{*}{$\begin{array}{c}\text { Industrial process } \\
\text { sources }\end{array}$} & Mining & 14.5 & 18.9 & 72.8 & 1.8 & 111.2 & 65.2 & 0.5 \\
\hline & Agricultural products processing & 0.3 & 0.1 & 0.3 & 62.7 & 97.7 & 70.5 & - \\
\hline & Food manufacturing & 0.1 & - & 0.1 & 0.6 & 5.6 & 3.9 & - \\
\hline & Beverage manufacturing & 0.1 & - & 0.1 & 104.1 & 0.1 & - & - \\
\hline & Papermaking & 17.7 & 0.4 & 30.7 & 8.7 & 10.0 & 8.0 & - \\
\hline & Petroleum refining & 9.7 & 4.5 & 629.8 & 156.4 & 23.1 & 22.3 & 2.0 \\
\hline & Coking & 34.7 & 17.6 & 241.3 & 1.3 & 17.6 & 13.2 & 0.1 \\
\hline & Chemical manufacturing & 93.6 & 39.6 & 474.1 & 801.2 & 21.1 & 15.2 & 45.0 \\
\hline & Rubber and plastic manufacturing & 0.6 & 0.4 & 1.0 & 254.7 & 0.1 & 0.1 & - \\
\hline & Non-metallic mineral manufacturing & 69.6 & 240.5 & 506.4 & 191.4 & 606.5 & 338.2 & - \\
\hline & Ferrous metal manufacturing & 77.5 & 105.2 & 383.5 & 32.6 & 91.2 & 69.9 & 0.3 \\
\hline & Non-ferrous metal manufacturing & 20.7 & 0.8 & 0.9 & 1.6 & 107.3 & 77.2 & - \\
\hline & Other miscellaneous sources & 6.7 & 4.0 & 19.2 & 55.4 & 4.4 & 2.8 & - \\
\hline \multirow{13}{*}{$\begin{array}{c}\text { Industrial solvent-use } \\
\text { sources }\end{array}$} & Textile & 1.5 & 1.0 & 2.1 & 102.4 & 0.3 & 0.2 & - \\
\hline & Leather tanning & - & - & - & 0.9 & - & - & - \\
\hline & Furniture and wood manufacturing & - & - & - & 363.4 & - & - & - \\
\hline & Package and printing & - & - & - & 51.0 & - & - & - \\
\hline & Pharmaceutical manufacturing & - & - & 0.1 & 103.2 & - & - & - \\
\hline & Shoemaking & - & - & - & 15.8 & - & - & - \\
\hline & Metal products & 0.2 & 0.1 & 0.1 & 77.6 & 40.7 & 24.4 & - \\
\hline & Machinery manufacturing & 0.3 & 0.8 & 1.0 & 135.5 & 19.4 & 13.2 & - \\
\hline & Railway equipment & 0.1 & 0.1 & 0.1 & 5.1 & 0.2 & 0.1 & - \\
\hline & Auto manufacturing & 0.2 & - & - & 71.0 & 1.0 & 0.6 & - \\
\hline & Shipbuilding & - & - & - & 20.8 & 0.2 & 0.2 & - \\
\hline & Appliance manufacturing & - & - & - & 272.2 & - & - & - \\
\hline & Electronic equipment & 0.3 & 0.1 & 0.2 & 100.8 & 0.1 & - & - \\
\hline \multirow{11}{*}{ Mobile sources } & Light-duty vehicles & 0.0 & 216.0 & 2694.3 & 434.9 & 17.7 & 17.4 & 28.2 \\
\hline & Light-duty trucks & 0.0 & 48.7 & 134.3 & 19.4 & 8.5 & 7.7 & 0.8 \\
\hline & Heavy-duty vehicles & 0.0 & 103.5 & 189.8 & 32.5 & 9.8 & 8.8 & 0.3 \\
\hline & Heavy-duty trucks & 0.0 & 571.7 & 201.4 & 63.3 & 49.8 & 44.9 & 0.0 \\
\hline & Taxi & 0.0 & 10.3 & 85.1 & 24.6 & 0.3 & 0.3 & 0.8 \\
\hline & Bus & 0.0 & 92.9 & 111.0 & 26.7 & 4.1 & 3.7 & 0.1 \\
\hline & Motorcycle & 0.0 & 5.4 & 175.0 & 228.9 & 0.8 & 0.8 & 1.1 \\
\hline & Construction machinery & 3.0 & 248.4 & 70.5 & 20.0 & 10.7 & 10.2 & 0.0 \\
\hline & Port machinery & 0.2 & 13.7 & 6.3 & 1.3 & 0.6 & 0.5 & 0.0 \\
\hline & Factory machinery & 0.5 & 48.8 & 19.6 & 4.7 & 2.7 & 2.5 & 0.0 \\
\hline & Agricultural machinery & 2.3 & 186.0 & 693.3 & 85.4 & 15.0 & 14.3 & 0.0 \\
\hline
\end{tabular}




\begin{tabular}{|c|c|c|c|c|c|c|c|c|}
\hline & Ground handling equipment & 0.1 & 3.7 & 2.4 & 0.4 & 0.1 & 0.1 & 0.0 \\
\hline & Marine & 114.9 & 298.8 & 21.3 & 7.8 & 15.9 & 14.6 & 0.1 \\
\hline & Aviation aircraft & 0.8 & 11.9 & 7.3 & 1.3 & 0.1 & 0.1 & 0.0 \\
\hline \multirow{2}{*}{ Dust sources } & Construction dust & - & - & - & - & 1480.8 & 302.2 & - \\
\hline & Road dust & - & - & - & - & 607.1 & 146.9 & - \\
\hline \multirow{2}{*}{$\begin{array}{c}\text { Oil storage and } \\
\text { transportation sources }\end{array}$} & Oil depot & - & - & - & 251.1 & - & - & - \\
\hline & Gas station & - & - & - & 44.5 & - & - & - \\
\hline \multirow{4}{*}{ Residential sources } & Human excretion & 58.9 & 28.1 & 438.8 & 15.1 & 54.0 & 48.3 & 3.4 \\
\hline & Residential combustion & - & - & - & 435.2 & - & - & - \\
\hline & Residential solvent-use & - & - & - & 20.5 & 10.2 & 8.2 & - \\
\hline & Catering & - & - & - & - & - & - & 82.2 \\
\hline \multirow{2}{*}{$\begin{array}{l}\text { Waste treatment and } \\
\text { disposal sources }\end{array}$} & Sewage treatment & - & - & - & 13.0 & - & - & 37.7 \\
\hline & Waste treatment & 3.4 & 30.1 & 1.2 & 8.7 & 1.4 & 1.4 & 17.3 \\
\hline \multirow{5}{*}{ Agricultural sources } & Biomass burning & 16.6 & 17.7 & 1587.9 & 122.1 & 104.6 & 97.3 & 5.4 \\
\hline & Fertilizer application & - & - & - & - & - & - & 32.5 \\
\hline & Soil background & - & - & - & - & - & - & 728.6 \\
\hline & Livestock and poultry breeding & - & - & - & - & - & - & 1467.6 \\
\hline & Total & 1552.5 & 3234.5 & 38507.1 & 4875.2 & 3770.5 & 1597.5 & 2466.7 \\
\hline
\end{tabular}

243

244

245 Table S6. Parameterization scheme and inputs for the WRF and CMAQ model.

\begin{tabular}{|c|c|c|c|}
\hline Model & Option & Configuration/Data source & Reference \\
\hline \multirow{7}{*}{ WRF } & Version & WRF-v3.9.1 & https://www2.mmm.ucar.edu/wrf/ \\
\hline & IC/BC condition & NCEP FNL $1^{\circ} \times 1^{\circ}$ & https://rda.ucar.edu/datasets/ds083.2/ \\
\hline & Microphysical Process & Purdue Lin Scheme & $\underline{\text { Lin, } 1983}$ \\
\hline & $\begin{array}{l}\text { Cumulus Convective } \\
\text { Scheme }\end{array}$ & Grell-3 Scheme & $\underline{\text { Grell and Dévényi, } 2002}$ \\
\hline & Road Process Scheme & Noah Scheme & $\underline{E k}, 2003$ \\
\hline & Boundary Layer Scheme & Yonsei University (YSU) Scheme & $\underline{\text { Hong, } 2006}$ \\
\hline & $\begin{array}{l}\text { Long-wave and Short- } \\
\text { wave radiation scheme }\end{array}$ & RRTM and Goddard radiation Scheme & $\frac{\text { Mlawer et al., 1997; Chou and }}{\text { Suarez, } 1999}$ \\
\hline \multirow{7}{*}{ CMAQ } & Version & CMAQ-v5.3.0 & https://www.cmascenter.org/cmaq/ \\
\hline & Domain & Three nested $\operatorname{grid}(36 / 12 / 4 \mathrm{~km})$ & - \\
\hline & Gas Chemical Mechanism & CB6r3 & $\frac{\text { http://www.camx.com/files/udaq_sno }}{\text { wchem_final_6aug15.pdf }}$ \\
\hline & $\begin{array}{l}\text { Aerosol Chemical } \\
\text { Mechanism }\end{array}$ & AERO6 & $\frac{\frac{\text { https://github.com/USEPA/CMAQ/bl }}{\text { ob/master/CCTM/src/MECHS/READ }}}{\underline{\text { ME.md }}}$ \\
\hline & $\begin{array}{l}\text { Anthropogenic emission } \\
\text { inventory }\end{array}$ & $\begin{array}{l}\text { MIX (2010) and } \operatorname{MEIC}(2016) \text { emissions } \\
\text { for } 36 \mathrm{~km}, 12 \mathrm{~km} \text { and area around the } \\
\text { YRD in } 4 \mathrm{~km} \text { domain }\end{array}$ & $\frac{\text { Li et al., } 2017}{\text { http://www.meicmodel.org/ }}$ \\
\hline & $\begin{array}{l}\text { Biogenic emission } \\
\text { inventory }\end{array}$ & MEGAN-v2.10 & $\frac{\text {;https://bai.ess.uci.edu/megan; Liu et }}{\underline{\text { al., } 2018}}$ \\
\hline & Emission processor & SMOKE-v46 & https://www.cmascenter.org/smoke/ \\
\hline
\end{tabular}


247 Table S7. The statistical results of model performance for the hourly concentrations in each city of 248 the YRD region in January and July 2017.

249 (a) $\mathrm{SO}_{2}$

Jan.

\begin{tabular}{|c|c|c|c|c|c|c|c|c|c|c|c|c|c|c|c|c|c|c|c|}
\hline \multirow[b]{2}{*}{ Province } & \multirow[b]{2}{*}{ City } & & & & & & & & & & \\
\hline & & $\begin{array}{l}\text { Data } \\
\text { pairs }\end{array}$ & $\begin{array}{c}\text { Obs. } \\
\left(\mu \mathrm{g} / \mathrm{m}^{3}\right)\end{array}$ & $\begin{array}{c}\text { Sim. } \\
\left(\mu \mathrm{g} / \mathrm{m}^{3}\right)\end{array}$ & $\begin{array}{l}\text { NMB } \\
(\%)\end{array}$ & $\begin{array}{l}\text { NME } \\
(\%)\end{array}$ & $\begin{array}{l}\text { MFB } \\
(\%)\end{array}$ & $\begin{array}{l}\text { MFE } \\
(\%)\end{array}$ & IOA & $\mathrm{R}$ & $\begin{array}{l}\text { Data } \\
\text { pairs }\end{array}$ & $\begin{array}{l}\text { Obs. } \\
\left(\mu \mathrm{g} / \mathrm{m}^{3}\right)\end{array}$ & $\begin{array}{c}\text { Sim. } \\
\left(\mu \mathrm{g} / \mathrm{m}^{3}\right)\end{array}$ & $\begin{array}{l}\text { NMB } \\
(\%)\end{array}$ & $\begin{array}{l}\text { NME } \\
(\%)\end{array}$ & $\begin{array}{l}\text { MFB } \\
(\%)\end{array}$ & $\begin{array}{l}\text { MFE } \\
(\%)\end{array}$ & IOA & $\mathrm{R}$ \\
\hline Shanghai & Shanghai & 624 & 18.0 & 10.2 & -43.5 & 51.2 & -69.0 & 74.3 & 0.6 & 0.5 & 741 & 11.3 & 7.0 & -37.6 & 60.1 & -65.3 & 83.7 & 0.4 & 0.1 \\
\hline \multirow{13}{*}{ Jiangsu } & Nanjing & 613 & 16.8 & 16.5 & -1.8 & 41.2 & -8.6 & 42.8 & 0.6 & 0.4 & 722 & 6.0 & 5.2 & -13.1 & 93.5 & -39.0 & 100.8 & 0.2 & -0.2 \\
\hline & Wuxi & 618 & 15.4 & 15.5 & 0.8 & 38.4 & -5.4 & 39.1 & 0.7 & 0.5 & 740 & 8.0 & 5.9 & -26.5 & 73.8 & -50.6 & 87.9 & 0.2 & -0.3 \\
\hline & Xuzhou & 606 & 33.9 & 36.9 & 8.9 & 47.8 & -2.4 & 44.1 & 0.6 & 0.4 & 740 & 13.0 & 16.0 & 23.4 & 76.5 & -8.5 & 67.9 & 0.3 & 0.1 \\
\hline & Changzhou & 622 & 16.3 & 18.8 & 15.5 & 44.9 & 7.3 & 40.7 & 0.6 & 0.4 & 701 & 16.2 & 7.6 & -53.0 & 61.4 & -86.2 & 93.1 & 0.5 & 0.2 \\
\hline & Suzhou & 623 & 17.1 & 14.2 & -17.0 & 37.7 & -29.7 & 46.8 & 0.7 & 0.6 & 618 & 8.5 & 7.9 & -7.2 & 58.4 & -23.6 & 63.7 & 0.4 & 0.1 \\
\hline & Nantong & 619 & 13.1 & 12.7 & -3.4 & 44.3 & -13.6 & 44.7 & 0.8 & 0.6 & 679 & 16.6 & 14.7 & -11.3 & 54.0 & -30.2 & 64.0 & 0.6 & 0.4 \\
\hline & Lianyungang & 601 & 31.3 & 12.5 & -60.2 & 61.3 & -92.4 & 93.7 & 0.5 & 0.5 & 741 & 5.5 & 3.0 & -45.5 & 65.6 & -79.6 & 96.1 & 0.3 & -0.1 \\
\hline & Huaian & 617 & 17.4 & 17.0 & -2.1 & 58.7 & -11.3 & 59.3 & 0.5 & 0.3 & 737 & 9.6 & 11.9 & 24.4 & 86.3 & -9.0 & 78.3 & 0.4 & 0.2 \\
\hline & Yancheng & 617 & 17.2 & 9.9 & -42.2 & 51.1 & -67.4 & 74.5 & 0.6 & 0.5 & 738 & 8.0 & 5.2 & -35.4 & 53.1 & -63.5 & 76.9 & 0.4 & 0.3 \\
\hline & Yangzhou & 624 & 14.9 & 14.0 & -6.0 & 41.6 & -16.3 & 44.6 & 0.7 & 0.5 & 691 & 18.1 & 8.1 & -54.9 & 62.1 & -89.4 & 96.0 & 0.5 & 0.3 \\
\hline & Zhenjiang & 621 & 12.0 & 19.9 & 65.9 & 83.3 & 43.9 & 57.7 & 0.6 & 0.5 & 741 & 16.9 & 12.7 & -25.1 & 58.2 & -38.4 & 70.2 & 0.5 & 0.1 \\
\hline & Taizhou & 500 & 14.4 & 10.8 & -24.9 & 47.7 & -45.1 & 61.7 & 0.6 & 0.4 & 740 & 12.4 & 8.9 & -28.2 & 72.6 & -28.8 & 94.0 & 0.4 & 0.1 \\
\hline & Suqian & 622 & 20.1 & 14.8 & -26.4 & 44.4 & -40.3 & 56.2 & 0.6 & 0.3 & 66 & 8.1 & 3.9 & -51.9 & 54.4 & -73.3 & 76.9 & 0.3 & 0.2 \\
\hline \multirow{11}{*}{ Zhejiang } & Hangzhou & 624 & 13.0 & 12.4 & -4.3 & 39.5 & -11.9 & 42.5 & 0.6 & 0.4 & 740 & 8.3 & 4.4 & -46.9 & 55.6 & -72.0 & 77.8 & 0.5 & 0.4 \\
\hline & Ningbo & 623 & 11.5 & 16.2 & 41.0 & 71.3 & 8.9 & 47.4 & 0.4 & 0.4 & 740 & 8.3 & 8.4 & 0.9 & 64.2 & -29.9 & 62.5 & 0.2 & 0.3 \\
\hline & Wenzhou & 575 & 6.6 & 8.0 & 21.6 & 65.6 & 24.9 & 64.3 & 0.6 & 0.4 & 741 & 10.4 & 6.0 & -42.7 & 52.9 & -67.8 & 77.7 & 0.5 & 0.4 \\
\hline & Jiaxing & 597 & 11.1 & 14.3 & 28.2 & 49.5 & 14.2 & 43.2 & 0.7 & 0.6 & 704 & 7.8 & 8.0 & 2.2 & 50.8 & -7.0 & 57.2 & 0.7 & 0.5 \\
\hline & Huzhou & 621 & 14.2 & 12.5 & -12.2 & 44.6 & -25.0 & 51.4 & 0.6 & 0.4 & 731 & 7.1 & 3.6 & -49.8 & 65.6 & -93.2 & 104.2 & 0.5 & 0.4 \\
\hline & Shaoxing & 623 & 12.1 & 15.1 & 24.5 & 56.7 & 15.2 & 49.7 & 0.5 & 0.3 & 678 & 12.1 & 8.1 & -32.8 & 52.1 & -43.2 & 66.2 & 0.5 & 0.2 \\
\hline & Jinhua & 623 & 12.4 & 9.5 & -23.3 & 46.7 & -25.3 & 58.7 & 0.6 & 0.4 & 734 & 7.4 & 3.7 & -50.0 & 60.5 & -76.2 & 85.9 & 0.5 & 0.3 \\
\hline & Quzhou & 622 & 12.0 & 21.0 & 75.8 & 108.0 & 45.7 & 68.4 & 0.3 & 0.1 & 722 & 15.5 & 27.4 & 76.9 & 132.5 & 29.2 & 90.2 & 0.4 & 0.2 \\
\hline & Zhoushan & 614 & 11.3 & 5.8 & -48.2 & 53.5 & -80.7 & 84.5 & 0.6 & 0.7 & 741 & 7.6 & 5.0 & -33.7 & 48.9 & -55.5 & 65.3 & 0.6 & 0.5 \\
\hline & Taizhou & 622 & 8.5 & 8.5 & 0.7 & 57.2 & 5.1 & 59.9 & 0.6 & 0.3 & 741 & 5.6 & 4.6 & -18.5 & 44.5 & -24.7 & 54.4 & 0.7 & 0.5 \\
\hline & Lishui & 621 & 9.3 & 6.2 & -32.9 & 50.4 & -54.2 & 69.3 & 0.5 & 0.3 & 712 & 7.0 & 1.5 & -78.1 & 78.3 & -136.3 & 136.4 & 0.4 & 0.3 \\
\hline \multirow{8}{*}{ Anhui } & Hefei & 624 & 18.1 & 14.8 & -18.2 & 41.7 & -21.7 & 46.5 & 0.6 & 0.3 & 732 & 5.1 & 4.3 & -15.6 & 53.9 & -24.7 & 61.8 & 0.5 & 0.2 \\
\hline & Wuhu & 614 & 20.6 & 15.4 & -25.3 & 48.6 & -35.4 & 56.0 & 0.4 & 0.1 & 737 & 11.5 & 9.4 & -18.2 & 60.1 & -25.7 & 71.0 & 0.6 & 0.3 \\
\hline & Bengbu & 618 & 17.4 & 14.5 & -16.8 & 55.7 & -19.8 & 58.5 & 0.5 & 0.1 & 739 & 8.5 & 4.5 & -47.3 & 56.3 & -74.4 & 84.4 & 0.5 & 0.2 \\
\hline & Huainan & 619 & 22.3 & 20.3 & -9.1 & 39.8 & -12.4 & 42.6 & 0.6 & 0.3 & 739 & 7.0 & 5.5 & -21.5 & 48.7 & -37.8 & 58.6 & 0.5 & 0.3 \\
\hline & Maanshan & 616 & 17.7 & 23.2 & 31.2 & 72.3 & 27.5 & 60.3 & 0.4 & 0.1 & 733 & 9.8 & 10.3 & 5.5 & 79.7 & 9.9 & 82.6 & 0.5 & 0.3 \\
\hline & Huaibei & 614 & 24.6 & 23.1 & -6.1 & 39.5 & -9.3 & 41.2 & 0.7 & 0.5 & 737 & 7.8 & 9.2 & 18.3 & 68.2 & -7.6 & 68.3 & 0.5 & 0.3 \\
\hline & Tongling & 564 & 23.5 & 23.2 & -1.3 & 62.1 & 0.9 & 60.2 & 0.5 & 0.2 & 731 & 19.2 & 9.6 & -49.9 & 63.3 & -72.4 & 87.3 & 0.5 & 0.2 \\
\hline & Anqing & 610 & 20.7 & 23.6 & 13.8 & 60.8 & 9.9 & 53.9 & 0.4 & 0.1 & 739 & 13.0 & 12.8 & -1.7 & 71.8 & -19.5 & 71.6 & 0.2 & -0.2 \\
\hline
\end{tabular}




\begin{tabular}{|c|c|c|c|c|c|c|c|c|c|c|c|c|c|c|c|c|c|c|c|}
\hline & Huangshan & 619 & 11.7 & 9.8 & -16.4 & 56.6 & -16.5 & 59.9 & 0.4 & 0.0 & 739 & 18.3 & 2.0 & -89.1 & 89.1 & -159.5 & 159.5 & 0.3 & 0.0 \\
\hline & Chuzhou & 621 & 14.7 & 14.3 & -2.7 & 55.5 & -14.8 & 57.5 & 0.5 & 0.2 & 737 & 12.3 & 5.0 & -59.2 & 67.5 & -87.4 & 97.0 & 0.4 & 0.0 \\
\hline & Fuyang & 603 & 15.0 & 27.2 & 81.4 & 101.1 & 45.6 & 63.3 & 0.3 & 0.2 & 739 & 13.4 & 12.0 & -10.6 & 72.1 & -31.9 & 79.5 & 0.4 & 0.0 \\
\hline & Suzhou & 591 & 24.9 & 19.9 & -20.4 & 52.5 & -15.6 & 57.4 & 0.6 & 0.3 & 703 & 14.8 & 4.7 & -68.3 & 71.8 & -98.2 & 104.0 & 0.4 & 0.1 \\
\hline & Lu'an & 622 & 16.5 & 13.5 & -18.2 & 46.9 & -27.2 & 54.5 & 0.5 & 0.2 & 738 & 8.4 & 3.2 & -62.0 & 64.4 & -93.5 & 96.2 & 0.4 & 0.0 \\
\hline & Bozhou & 615 & 29.8 & 19.1 & -35.8 & 51.4 & -48.3 & 64.8 & 0.5 & 0.2 & 723 & 9.4 & 4.0 & -57.3 & 59.7 & -89.4 & 91.8 & 0.4 & 0.2 \\
\hline & Chizhou & 604 & 23.8 & 19.6 & -17.7 & 50.6 & -23.8 & 54.6 & 0.5 & 0.2 & 739 & 18.9 & 3.8 & -79.8 & 81.3 & -129.6 & 131.6 & 0.4 & -0.1 \\
\hline & Xuancheng & 612 & 21.0 & 16.0 & -24.0 & 46.3 & -34.9 & 56.1 & 0.3 & -0.1 & 741 & 14.1 & 2.9 & -79.5 & 80.4 & -130.7 & 132.2 & 0.4 & 0.2 \\
\hline 250 & (b) $\mathrm{NO}_{2}$ & & & & & & & & & & & & & & & & & & \\
\hline \multirow[b]{2}{*}{ Province } & \multirow[b]{2}{*}{ City } & \multicolumn{9}{|c|}{ Jan } & \multicolumn{9}{|c|}{ Jul } \\
\hline & & $\begin{array}{l}\text { Data } \\
\text { pairs }\end{array}$ & $\begin{array}{c}\text { Obs. } \\
\left(\mu \mathrm{g} / \mathrm{m}^{3}\right)\end{array}$ & $\begin{array}{c}\text { Sim. } \\
\left(\mu \mathrm{g} / \mathrm{m}^{3}\right)\end{array}$ & $\begin{array}{l}\text { NMB } \\
(\%)\end{array}$ & $\begin{array}{l}\text { NME } \\
(\%)\end{array}$ & $\begin{array}{c}\text { MFB } \\
(\%)\end{array}$ & $\begin{array}{l}\text { MFE } \\
(\%)\end{array}$ & IOA & $\mathrm{R}$ & $\begin{array}{l}\text { Data } \\
\text { pairs }\end{array}$ & $\begin{array}{c}\text { Obs. } \\
\left(\mu \mathrm{g} / \mathrm{m}^{3}\right)\end{array}$ & $\begin{array}{c}\text { Sim. } \\
\left(\mu \mathrm{g} / \mathrm{m}^{3}\right)\end{array}$ & $\begin{array}{c}\text { NMB } \\
(\%)\end{array}$ & $\begin{array}{c}\text { NME } \\
(\%)\end{array}$ & $\begin{array}{c}\text { MFB } \\
(\%)\end{array}$ & $\begin{array}{l}\text { MFE } \\
(\%)\end{array}$ & IOA & $\mathrm{R}$ \\
\hline Shanghai & Shanghai & 624 & 44.7 & 50.6 & 13.2 & 38.1 & 11.9 & 35.8 & 0.7 & 0.5 & 738 & 30.1 & 42.7 & 41.7 & 63.7 & 24.9 & 47.1 & 0.6 & 0.5 \\
\hline \multirow{13}{*}{ Jiangsu } & Nanjing & 613 & 36.5 & 45.6 & 25.0 & 48.6 & 19.7 & 45.4 & 0.7 & 0.5 & 723 & 25.7 & 24.6 & -4.2 & 62.1 & -18.2 & 64.8 & 0.6 & 0.3 \\
\hline & Wuxi & 615 & 58.5 & 51.6 & -11.8 & 31.3 & -9.1 & 34.6 & 0.7 & 0.6 & 740 & 26.7 & 31.4 & 17.7 & 56.6 & 6.5 & 45.9 & 0.6 & 0.4 \\
\hline & Xuzhou & 620 & 54.2 & 49.8 & -8.1 & 28.5 & -7.3 & 31.2 & 0.8 & 0.6 & 740 & 22.0 & 24.6 & 11.7 & 46.8 & 15.1 & 44.1 & 0.7 & 0.5 \\
\hline & Changzhou & 622 & 42.5 & 50.9 & 19.7 & 41.6 & 19.2 & 42.3 & 0.7 & 0.5 & 704 & 26.3 & 29.7 & 13.1 & 59.5 & 2.1 & 52.2 & 0.6 & 0.3 \\
\hline & Suzhou & 623 & 50.3 & 50.6 & 0.6 & 31.6 & 0.8 & 33.6 & 0.8 & 0.6 & 618 & 30.3 & 33.4 & 10.5 & 56.1 & -1.5 & 51.5 & 0.5 & 0.2 \\
\hline & Nantong & 619 & 32.2 & 36.2 & 12.5 & 43.5 & 9.7 & 42.4 & 0.8 & 0.6 & 678 & 45.5 & 43.5 & -4.4 & 51.5 & -9.0 & 55.5 & 0.6 & 0.4 \\
\hline & Lianyungang & 621 & 39.7 & 34.0 & -14.4 & 41.4 & -8.7 & 46.1 & 0.7 & 0.5 & 741 & 17.4 & 18.2 & 4.5 & 50.5 & -4.9 & 46.8 & 0.5 & 0.3 \\
\hline & Huaian & 620 & 29.8 & 31.9 & 6.9 & 45.4 & 2.2 & 42.0 & 0.7 & 0.5 & 738 & 23.3 & 33.0 & 41.6 & 66.7 & 29.8 & 53.0 & 0.5 & 0.3 \\
\hline & Yancheng & 617 & 32.8 & 20.8 & -36.7 & 50.6 & -48.2 & 66.4 & 0.7 & 0.5 & 739 & 21.7 & 16.9 & -22.0 & 48.9 & -42.6 & 62.1 & 0.6 & 0.4 \\
\hline & Yangzhou & 619 & 43.8 & 34.2 & -22.0 & 38.4 & -28.5 & 43.9 & 0.7 & 0.6 & 689 & 29.1 & 28.1 & -3.5 & 49.5 & -19.6 & 53.5 & 0.7 & 0.5 \\
\hline & Zhenjiang & 621 & 42.6 & 38.4 & -9.9 & 35.3 & -14.6 & 39.4 & 0.8 & 0.6 & 740 & 29.3 & 30.5 & 4.2 & 52.2 & 4.6 & 52.5 & 0.6 & 0.4 \\
\hline & Taizhou & 500 & 27.1 & 28.5 & 5.2 & 43.9 & -0.3 & 40.2 & 0.7 & 0.5 & 740 & 24.8 & 29.4 & 18.7 & 67.4 & 5.0 & 58.6 & 0.5 & 0.3 \\
\hline & Suqian & 622 & 39.1 & 36.0 & -8.1 & 36.8 & -11.6 & 39.8 & 0.7 & 0.5 & 66 & 25.5 & 20.8 & -18.2 & 46.2 & -29.0 & 54.9 & 0.6 & 0.4 \\
\hline \multirow{11}{*}{ Zhejiang } & Hangzhou & 624 & 44.6 & 47.5 & 6.7 & 38.1 & 9.3 & 39.8 & 0.7 & 0.5 & 740 & 26.6 & 32.5 & 22.1 & 56.4 & 14.8 & 46.7 & 0.6 & 0.3 \\
\hline & Ningbo & 623 & 41.8 & 40.5 & -3.2 & 37.5 & -3.5 & 40.5 & 0.8 & 0.6 & 740 & 31.3 & 34.8 & 11.1 & 43.1 & 11.4 & 38.3 & 0.7 & 0.6 \\
\hline & Wenzhou & 574 & 31.1 & 30.9 & -0.7 & 42.8 & -1.1 & 42.5 & 0.6 & 0.4 & 741 & 25.9 & 29.5 & 14.3 & 44.4 & 3.8 & 38.2 & 0.6 & 0.5 \\
\hline & Jiaxing & 597 & 35.8 & 38.0 & 6.3 & 43.8 & 11.6 & 44.1 & 0.7 & 0.4 & 704 & 26.4 & 26.0 & -1.8 & 48.6 & -8.7 & 48.5 & 0.7 & 0.5 \\
\hline & Huzhou & 621 & 37.1 & 29.7 & -20.0 & 46.2 & -22.4 & 52.4 & 0.6 & 0.4 & 731 & 27.4 & 10.7 & -61.0 & 70.4 & -92.1 & 103.8 & 0.4 & 0.1 \\
\hline & Shaoxing & 623 & 45.7 & 36.9 & -19.2 & 36.7 & -20.3 & 42.9 & 0.7 & 0.5 & 670 & 19.2 & 16.9 & -12.0 & 40.0 & -17.9 & 41.3 & 0.6 & 0.4 \\
\hline & Jinhua & 623 & 32.8 & 29.7 & -9.2 & 37.2 & -8.1 & 41.4 & 0.7 & 0.5 & 732 & 19.0 & 13.5 & -29.1 & 52.0 & -45.0 & 61.9 & 0.5 & 0.3 \\
\hline & Quzhou & 623 & 34.7 & 46.6 & 34.2 & 66.2 & 21.2 & 52.6 & 0.3 & 0.1 & 730 & 23.9 & 45.2 & 89.1 & 116.9 & 51.7 & 76.3 & 0.3 & -0.1 \\
\hline & Zhoushan & 614 & 13.0 & 15.7 & 20.7 & 54.3 & 14.5 & 48.6 & 0.7 & 0.6 & 741 & 15.4 & 18.6 & 21.1 & 51.3 & 12.2 & 43.0 & 0.7 & 0.5 \\
\hline & Taizhou & 622 & 24.9 & 27.2 & 9.5 & 45.5 & 11.0 & 42.7 & 0.6 & 0.4 & 741 & 11.9 & 17.3 & 45.2 & 66.4 & 30.9 & 47.2 & 0.7 & 0.6 \\
\hline & Lishui & 621 & 21.0 & 13.7 & -34.6 & 46.7 & -36.8 & 57.7 & 0.6 & 0.5 & 712 & 14.6 & 7.5 & -48.5 & 51.7 & -60.7 & 65.4 & 0.6 & 0.4 \\
\hline \multirow{2}{*}{ Anhui } & Hefei & 624 & 44.8 & 48.0 & 7.3 & 35.0 & 11.4 & 35.6 & 0.7 & 0.6 & 732 & 30.2 & 32.4 & 7.1 & 39.3 & 3.6 & 36.1 & 0.7 & 0.5 \\
\hline & Wuhu & 613 & 42.6 & 32.7 & -23.3 & 44.2 & -19.8 & 48.4 & 0.7 & 0.5 & 740 & 31.4 & 23.1 & -26.5 & 49.7 & -23.9 & 57.2 & 0.7 & 0.5 \\
\hline
\end{tabular}




\begin{tabular}{|c|c|c|c|c|c|c|c|c|c|c|c|c|c|c|c|c|c|c|}
\hline Bengbu & 621 & 34.6 & 31.5 & -8.9 & 45.7 & -0.5 & 52.4 & 0.7 & 0.5 & 739 & 20.7 & 15.7 & -24.3 & 46.9 & -30.6 & 56.8 & 0.7 & 0.5 \\
\hline Huainan & 622 & 25.8 & 40.0 & 54.6 & 70.0 & 46.9 & 57.9 & 0.6 & 0.4 & 739 & 17.6 & 17.3 & -1.9 & 52.9 & -8.4 & 50.1 & 0.4 & 0.2 \\
\hline Maanshan & 616 & 36.0 & 44.8 & 24.5 & 49.4 & 25.0 & 46.8 & 0.6 & 0.4 & 737 & 23.5 & 23.4 & -0.5 & 53.3 & -1.8 & 51.9 & 0.6 & 0.3 \\
\hline Huaibei & 616 & 57.9 & 42.0 & -27.4 & 40.0 & -29.9 & 51.6 & 0.6 & 0.5 & 737 & 23.1 & 22.0 & -5.0 & 43.1 & -5.7 & 42.4 & 0.6 & 0.4 \\
\hline Tongling & 624 & 40.0 & 35.4 & -11.5 & 42.6 & -10.1 & 47.4 & 0.7 & 0.4 & 741 & 31.1 & 14.2 & -54.4 & 60.0 & -70.1 & 77.6 & 0.6 & 0.5 \\
\hline Anqing & 619 & 40.8 & 30.1 & -26.2 & 41.4 & -24.1 & 44.6 & 0.6 & 0.4 & 741 & 29.3 & 16.4 & -43.9 & 56.8 & -40.4 & 62.2 & 0.5 & 0.3 \\
\hline Huangshan & 622 & 18.0 & 13.0 & -28.0 & 43.2 & -33.4 & 49.2 & 0.5 & 0.3 & 739 & 20.7 & 6.9 & -66.5 & 66.7 & -103.9 & 104.1 & 0.4 & 0.3 \\
\hline Chuzhou & 619 & 38.5 & 32.5 & -15.6 & 41.7 & -21.9 & 47.2 & 0.7 & 0.5 & 739 & 23.4 & 15.3 & -34.6 & 54.8 & -45.5 & 62.3 & 0.5 & 0.3 \\
\hline Fuyang & 603 & 40.1 & 42.1 & 5.2 & 33.8 & 2.7 & 33.4 & 0.7 & 0.5 & 739 & 21.3 & 18.0 & -15.4 & 39.5 & -21.1 & 43.1 & 0.6 & 0.3 \\
\hline Suzhou & 622 & 43.9 & 41.4 & -5.8 & 35.9 & 0.0 & 38.7 & 0.8 & 0.6 & 718 & 23.9 & 22.6 & -5.3 & 39.6 & -5.4 & 40.5 & 0.7 & 0.5 \\
\hline Liuan & 623 & 39.7 & 28.2 & -28.9 & 40.9 & -37.1 & 50.7 & 0.6 & 0.5 & 737 & 22.1 & 14.4 & -34.7 & 49.8 & -50.9 & 66.9 & 0.5 & 0.3 \\
\hline Bozhou & 618 & 30.6 & 37.3 & 21.9 & 47.1 & 25.8 & 47.6 & 0.7 & 0.6 & 723 & 12.0 & 13.3 & 10.1 & 50.7 & 4.9 & 47.2 & 0.6 & 0.3 \\
\hline Chizhou & 622 & 39.2 & 27.1 & -30.7 & 44.8 & -39.3 & 53.9 & 0.6 & 0.4 & 739 & 21.9 & 8.5 & -61.1 & 62.7 & -88.1 & 89.9 & 0.4 & 0.3 \\
\hline Xuancheng & 612 & 22.8 & 26.8 & 17.7 & 60.9 & 12.8 & 54.1 & 0.6 & 0.3 & 741 & 25.7 & 11.8 & -54.3 & 63.8 & -74.0 & 91.0 & 0.4 & 0.0 \\
\hline
\end{tabular}

251

(c) $\mathrm{O}_{3}$

Jan

Jul

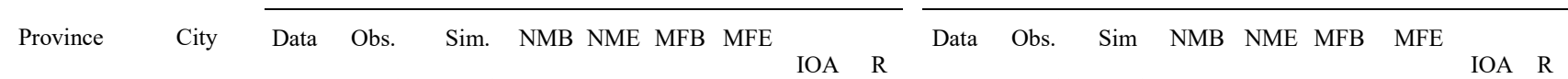
$\begin{array}{llllllllllllllll}\text { pairs } & \left(\mu \mathrm{g} / \mathrm{m}^{3}\right) & \left(\mu \mathrm{g} / \mathrm{m}^{3}\right) & (\%) & (\%) & (\%) & (\%) & & & \text { pairs } & \left(\mu \mathrm{g} / \mathrm{m}^{3}\right) & \left(\mu \mathrm{g} / \mathrm{m}^{3}\right) & (\%) & (\%) & (\%) & (\%)\end{array}$

\begin{tabular}{|c|c|c|c|c|c|c|c|c|c|c|c|c|c|c|c|c|c|c|c|}
\hline Shanghai & Shanghai & 624 & 61.4 & 45.6 & -25.7 & 37.4 & -46.5 & 61.7 & 0.7 & 0.6 & 677 & 90.5 & 62.4 & -31.0 & 40.4 & -57.3 & 71.3 & 0.8 & 0.8 \\
\hline & Nanjing & 613 & 63.3 & 40.9 & -35.4 & 49.7 & -55.3 & 76.5 & 0.6 & 0.4 & 713 & 85.5 & 81.8 & -4.3 & 43.4 & -10.1 & 53.1 & 0.8 & 0.6 \\
\hline & Wuxi & 618 & 39.6 & 39.3 & -0.8 & 46.1 & -21.8 & 66.6 & 0.8 & 0.6 & 729 & 87.8 & 72.7 & -17.1 & 34.1 & -25.9 & 52.0 & 0.9 & 0.8 \\
\hline & Xuzhou & 622 & 30.0 & 33.1 & 10.4 & 51.6 & -13.0 & 75.6 & 0.8 & 0.7 & 740 & 84.9 & 93.8 & 10.5 & 35.0 & 18.1 & 40.0 & 0.8 & 0.7 \\
\hline & Changzhou & 622 & 40.9 & 39.8 & -2.6 & 52.1 & -30.2 & 73.9 & 0.7 & 0.5 & 699 & 81.1 & 71.3 & -12.0 & 38.4 & -20.6 & 56.3 & 0.8 & 0.7 \\
\hline & Suzhou & 623 & 41.8 & 41.5 & -0.6 & 47.4 & -29.5 & 64.2 & 0.7 & 0.6 & 616 & 85.4 & 74.6 & -12.6 & 38.3 & -30.1 & 55.5 & 0.8 & 0.7 \\
\hline & Nantong & 619 & 62.4 & 53.7 & -13.9 & 30.8 & -28.0 & 46.5 & 0.7 & 0.6 & 657 & 85.1 & 72.7 & -14.6 & 37.0 & -33.4 & 62.1 & 0.9 & 0.8 \\
\hline \multirow[t]{10}{*}{ Jiangsu } & Lianyungang & 620 & 47.0 & 49.7 & 5.9 & 38.9 & 12.0 & 52.8 & 0.8 & 0.6 & 736 & 65.9 & 94.1 & 42.8 & 53.4 & 36.3 & 47.6 & 0.7 & 0.6 \\
\hline & Huaian & 621 & 46.9 & 52.7 & 12.3 & 38.9 & 8.4 & 48.5 & 0.7 & 0.6 & 717 & 72.2 & 73.7 & 2.1 & 40.1 & 10.8 & 49.2 & 0.8 & 0.6 \\
\hline & Yancheng & 611 & 57.2 & 64.8 & 13.4 & 34.5 & 12.5 & 41.5 & 0.7 & 0.5 & 739 & 60.0 & 81.5 & 36.0 & 52.5 & 32.5 & 50.4 & 0.7 & 0.6 \\
\hline & Yangzhou & 624 & 49.7 & 52.7 & 6.0 & 35.8 & -2.9 & 51.1 & 0.8 & 0.6 & 646 & 82.1 & 72.4 & -11.8 & 42.0 & -13.2 & 61.6 & 0.8 & 0.7 \\
\hline & Zhenjiang & 621 & 49.8 & 49.8 & 0.0 & 36.6 & -15.0 & 54.1 & 0.8 & 0.6 & 738 & 64.9 & 70.5 & 8.6 & 41.6 & 8.5 & 59.1 & 0.9 & 0.8 \\
\hline & Taizhou & 499 & 51.1 & 59.1 & 15.6 & 35.5 & 4.6 & 40.0 & 0.7 & 0.5 & 728 & 77.4 & 73.8 & -4.7 & 39.9 & -14.4 & 57.2 & 0.9 & 0.7 \\
\hline & Suqian & 612 & 42.7 & 47.6 & 11.4 & 45.0 & 1.7 & 56.7 & 0.7 & 0.6 & 66 & 66.3 & 71.6 & 8.0 & 30.8 & 13.1 & 34.6 & 0.9 & 0.7 \\
\hline & Hangzhou & 624 & 38.2 & 48.7 & 27.5 & 63.9 & 15.0 & 73.1 & 0.7 & 0.6 & 700 & 70.1 & 62.1 & -11.4 & 45.8 & -5.5 & 61.9 & 0.8 & 0.7 \\
\hline & Ningbo & 623 & 50.6 & 60.4 & 19.3 & 40.6 & 19.6 & 51.8 & 0.8 & 0.6 & 717 & 69.7 & 59.6 & -14.4 & 34.0 & -16.3 & 48.0 & 0.9 & 0.8 \\
\hline & Wenzhou & 575 & 49.1 & 69.2 & 41.0 & 56.9 & 39.9 & 57.4 & 0.7 & 0.6 & 725 & 61.3 & 53.1 & -13.4 & 36.8 & -12.9 & 47.4 & 0.9 & 0.8 \\
\hline \multirow[t]{4}{*}{ Zhejiang } & Jiaxing & 574 & 43.9 & 55.2 & 25.8 & 52.8 & 24.2 & 58.7 & 0.7 & 0.5 & 689 & 91.0 & 79.1 & -13.2 & 29.7 & -12.9 & 46.8 & 0.9 & 0.8 \\
\hline & Huzhou & 621 & 45.4 & 61.1 & 34.7 & 59.8 & 38.1 & 64.3 & 0.7 & 0.4 & 717 & 68.6 & 78.8 & 14.8 & 49.6 & 42.6 & 67.0 & 0.8 & 0.8 \\
\hline & Shaoxing & 623 & 37.8 & 58.2 & 54.1 & 71.7 & 43.1 & 71.1 & 0.7 & 0.6 & 649 & 90.5 & 76.0 & -16.0 & 28.3 & -18.0 & 35.3 & 0.9 & 0.8 \\
\hline & Jinhua & 614 & 36.0 & 65.6 & 82.1 & 92.4 & 64.0 & 76.5 & 0.6 & 0.6 & 722 & 73.9 & 64.4 & -12.8 & 32.8 & -4.8 & 42.3 & 0.9 & 0.8 \\
\hline
\end{tabular}




\begin{tabular}{|c|c|c|c|c|c|c|c|c|c|c|c|c|c|c|c|c|c|c|c|}
\hline & Quzhou & 623 & 39.3 & 51.6 & 31.5 & 64.2 & 28.2 & 67.4 & 0.7 & 0.4 & 726 & 60.1 & 34.7 & -42.2 & 59.4 & -31.0 & 80.5 & 0.6 & 0.6 \\
\hline & Zhoushan & 614 & 76.4 & 84.6 & 10.8 & 23.7 & 11.3 & 25.7 & 0.7 & 0.6 & 739 & 61.9 & 51.8 & -16.2 & 33.9 & -23.2 & 43.4 & 0.8 & 0.7 \\
\hline & Taizhou & 622 & 58.5 & 70.6 & 20.7 & 42.2 & 22.8 & 45.5 & 0.7 & 0.5 & 741 & 63.6 & 59.0 & -7.3 & 32.0 & -14.8 & 41.1 & 0.9 & 0.8 \\
\hline & Lishui & 621 & 53.8 & 84.9 & 57.7 & 70.2 & 54.3 & 63.7 & 0.6 & 0.5 & 712 & 65.8 & 69.3 & 5.4 & 36.9 & 24.7 & 47.8 & 0.8 & 0.8 \\
\hline \multirow{16}{*}{ Anhui } & Hefei & 624 & 39.3 & 38.1 & -3.0 & 48.0 & -19.2 & 66.0 & 0.8 & 0.6 & 719 & 86.3 & 75.4 & -12.6 & 37.5 & -10.9 & 48.3 & 0.8 & 0.7 \\
\hline & Wuhu & 616 & 54.3 & 53.5 & -1.3 & 45.2 & -16.1 & 55.2 & 0.5 & 0.3 & 667 & 76.9 & 72.2 & -6.1 & 45.2 & 17.0 & 61.1 & 0.8 & 0.7 \\
\hline & Bengbu & 621 & 46.9 & 52.2 & 11.3 & 44.7 & 7.7 & 56.9 & 0.8 & 0.6 & 739 & 76.9 & 89.4 & 16.3 & 33.9 & 20.2 & 37.4 & 0.9 & 0.8 \\
\hline & Huainan & 622 & 44.5 & 44.8 & 0.6 & 46.4 & 0.0 & 60.2 & 0.7 & 0.5 & 736 & 74.4 & 84.0 & 12.9 & 36.7 & 18.5 & 41.2 & 0.8 & 0.6 \\
\hline & Maanshan & 604 & 39.1 & 42.7 & 9.1 & 52.8 & -4.7 & 64.9 & 0.7 & 0.5 & 715 & 79.3 & 79.7 & 0.5 & 39.3 & 14.5 & 51.9 & 0.9 & 0.8 \\
\hline & Huaibei & 618 & 35.0 & 39.9 & 14.2 & 52.9 & 4.0 & 67.1 & 0.8 & 0.6 & 737 & 78.5 & 95.3 & 21.4 & 40.1 & 27.1 & 43.7 & 0.8 & 0.7 \\
\hline & Tongling & 624 & 48.1 & 53.9 & 12.1 & 56.3 & 4.0 & 63.8 & 0.6 & 0.4 & 741 & 67.4 & 82.5 & 22.4 & 44.9 & 27.6 & 46.5 & 0.8 & 0.6 \\
\hline & Anqing & 618 & 54.2 & 60.7 & 12.0 & 49.6 & 13.5 & 52.3 & 0.6 & 0.3 & 737 & 68.1 & 80.7 & 18.6 & 36.7 & 20.9 & 38.2 & 0.8 & 0.7 \\
\hline & Huangshan & 621 & 62.9 & 85.8 & 36.3 & 50.1 & 31.3 & 44.3 & 0.6 & 0.4 & 739 & 52.8 & 69.6 & 31.8 & 42.9 & 34.7 & 41.7 & 0.8 & 0.7 \\
\hline & Chuzhou & 621 & 72.6 & 52.3 & -27.9 & 40.7 & -42.2 & 58.7 & 0.6 & 0.4 & 739 & 88.0 & 89.5 & 1.7 & 28.7 & -3.9 & 35.1 & 0.8 & 0.7 \\
\hline & Fuyang & 602 & 34.5 & 40.9 & 18.4 & 55.2 & 4.7 & 66.1 & 0.8 & 0.6 & 739 & 59.4 & 84.4 & 42.0 & 47.8 & 40.0 & 46.3 & 0.8 & 0.7 \\
\hline & Suzhou & 622 & 38.8 & 40.7 & 4.8 & 42.7 & -11.5 & 59.9 & 0.8 & 0.7 & 731 & 85.8 & 86.0 & 0.2 & 31.3 & 5.6 & 36.6 & 0.8 & 0.7 \\
\hline & Liuan & 624 & 49.3 & 55.2 & 12.1 & 49.1 & 11.1 & 55.7 & 0.7 & 0.5 & 736 & 70.1 & 83.7 & 19.3 & 38.7 & 23.7 & 40.7 & 0.8 & 0.6 \\
\hline & Bozhou & 618 & 42.9 & 43.0 & 0.3 & 38.8 & -13.2 & 51.6 & 0.8 & 0.7 & 722 & 88.8 & 97.6 & 9.9 & 31.0 & 14.0 & 33.4 & 0.8 & 0.6 \\
\hline & Chizhou & 621 & 40.6 & 63.1 & 55.3 & 72.2 & 40.7 & 63.7 & 0.6 & 0.4 & 739 & 79.9 & 80.3 & 0.5 & 24.5 & 0.4 & 26.4 & 0.8 & 0.7 \\
\hline & Xuancheng & 612 & 37.6 & 62.7 & 66.6 & 92.8 & 37.0 & 67.8 & 0.3 & 0.0 & 741 & 64.8 & 71.1 & 9.6 & 33.4 & 13.6 & 37.0 & 0.8 & 0.7 \\
\hline
\end{tabular}

252 (d) $\mathrm{PM}_{2.5}$

\begin{tabular}{|c|c|c|c|c|c|c|c|c|c|c|c|c|c|c|c|c|c|c|c|}
\hline \multirow[b]{2}{*}{ Province } & \multirow[b]{2}{*}{ City } & \multicolumn{9}{|c|}{ Jan } & \multicolumn{9}{|c|}{ Jul } \\
\hline & & $\begin{array}{l}\text { Data } \\
\text { pairs }\end{array}$ & $\begin{array}{c}\text { Obs. } \\
\left(\mu \mathrm{g} / \mathrm{m}^{3}\right)\end{array}$ & $\begin{array}{c}\text { Sim. } \\
\left(\mu \mathrm{g} / \mathrm{m}^{3}\right)\end{array}$ & $\begin{array}{c}\text { NMB } \\
(\%)\end{array}$ & $\begin{array}{c}\text { NME } \\
(\%)\end{array}$ & $\begin{array}{c}\text { MFB } \\
(\%)\end{array}$ & $\begin{array}{c}\text { MFE } \\
(\%)\end{array}$ & IOA & $\mathrm{R}$ & $\begin{array}{l}\text { Data } \\
\text { pairs }\end{array}$ & $\begin{array}{c}\text { Obs. } \\
\left(\mu \mathrm{g} / \mathrm{m}^{3}\right)\end{array}$ & $\begin{array}{c}\text { Sim. } \\
\left(\mu \mathrm{g} / \mathrm{m}^{3}\right)\end{array}$ & $\begin{array}{c}\text { NMB } \\
(\%)\end{array}$ & $\begin{array}{l}\text { NME } \\
(\%)\end{array}$ & $\begin{array}{c}\text { MFB } \\
(\%)\end{array}$ & $\begin{array}{c}\text { MFE } \\
(\%)\end{array}$ & IOA & $\mathrm{R}$ \\
\hline Shanghai & Shanghai & 616 & 41.6 & 36.6 & -12.0 & 37.7 & -4.7 & 37.8 & 0.8 & 0.6 & 732 & 30.7 & 30.2 & -1.8 & 40.5 & -10.2 & 40.9 & 0.7 & 0.6 \\
\hline \multirow{13}{*}{ Jiangsu } & Nanjing & 611 & 46.4 & 46.3 & -0.2 & 43.2 & 4.3 & 47.7 & 0.7 & 0.5 & 628 & 18.8 & 22.8 & 21.5 & 54.5 & 17.6 & 54.1 & 0.7 & 0.6 \\
\hline & Wuxi & 617 & 48.2 & 44.5 & -7.7 & 40.0 & -6.5 & 42.1 & 0.7 & 0.5 & 734 & 25.0 & 24.9 & -0.4 & 51.8 & -9.6 & 51.6 & 0.7 & 0.5 \\
\hline & Xuzhou & 597 & 83.4 & 61.7 & -26.0 & 35.6 & -29.2 & 40.5 & 0.7 & 0.6 & 735 & 34.3 & 27.1 & -21.0 & 45.1 & -21.8 & 51.9 & 0.6 & 0.4 \\
\hline & Changzhou & 620 & 58.0 & 48.2 & -17.0 & 37.6 & -16.4 & 42.8 & 0.7 & 0.5 & 704 & 28.3 & 24.7 & -12.8 & 42.3 & -18.5 & 48.4 & 0.8 & 0.6 \\
\hline & Suzhou & 622 & 53.2 & 45.2 & -15.0 & 36.7 & -9.1 & 39.2 & 0.8 & 0.6 & 617 & 23.7 & 30.3 & 28.0 & 57.9 & 10.6 & 43.5 & 0.5 & 0.5 \\
\hline & Nantong & 618 & 53.4 & 38.3 & -28.3 & 38.7 & -27.8 & 45.7 & 0.8 & 0.7 & 681 & 34.8 & 30.4 & -12.7 & 38.6 & -15.6 & 43.4 & 0.7 & 0.5 \\
\hline & Lianyungang & 618 & 52.8 & 41.8 & -20.8 & 41.6 & -23.3 & 48.2 & 0.7 & 0.6 & 741 & 33.5 & 17.7 & -47.2 & 54.9 & -59.3 & 70.5 & 0.5 & 0.3 \\
\hline & Huaian & 621 & 58.6 & 48.3 & -17.6 & 44.3 & -13.4 & 51.1 & 0.7 & 0.5 & 737 & 33.9 & 24.6 & -27.5 & 40.0 & -38.9 & 50.8 & 0.5 & 0.3 \\
\hline & Yancheng & 612 & 45.8 & 34.5 & -24.6 & 47.1 & -18.1 & 53.4 & 0.7 & 0.6 & 730 & 28.0 & 23.2 & -17.2 & 50.1 & -25.0 & 57.5 & 0.6 & 0.4 \\
\hline & Yangzhou & 612 & 63.4 & 49.0 & -22.8 & 39.2 & -19.8 & 46.6 & 0.7 & 0.6 & 691 & 34.7 & 26.7 & -23.2 & 39.3 & -33.1 & 47.5 & 0.7 & 0.6 \\
\hline & Zhenjiang & 621 & 58.2 & 43.5 & -25.3 & 35.1 & -30.1 & 41.3 & 0.7 & 0.6 & 741 & 36.2 & 24.3 & -32.7 & 44.1 & -47.1 & 58.5 & 0.7 & 0.5 \\
\hline & Taizhou & 494 & 53.0 & 37.8 & -28.8 & 42.2 & -29.7 & 48.2 & 0.7 & 0.5 & 736 & 32.7 & 28.9 & -11.6 & 43.1 & -16.9 & 47.1 & 0.6 & 0.4 \\
\hline & Suqian & 622 & 60.5 & 47.5 & -21.4 & 37.5 & -22.0 & 44.4 & 0.8 & 0.7 & 65 & 28.4 & 16.1 & -43.4 & 48.0 & -51.0 & 57.5 & 0.4 & 0.0 \\
\hline Zhejiang & Hangzhou & 624 & 51.5 & 43.4 & -15.7 & 39.3 & -16.8 & 43.4 & 0.7 & 0.5 & 740 & 26.3 & 19.7 & -25.2 & 33.8 & -33.1 & 42.6 & 0.8 & 0.8 \\
\hline
\end{tabular}




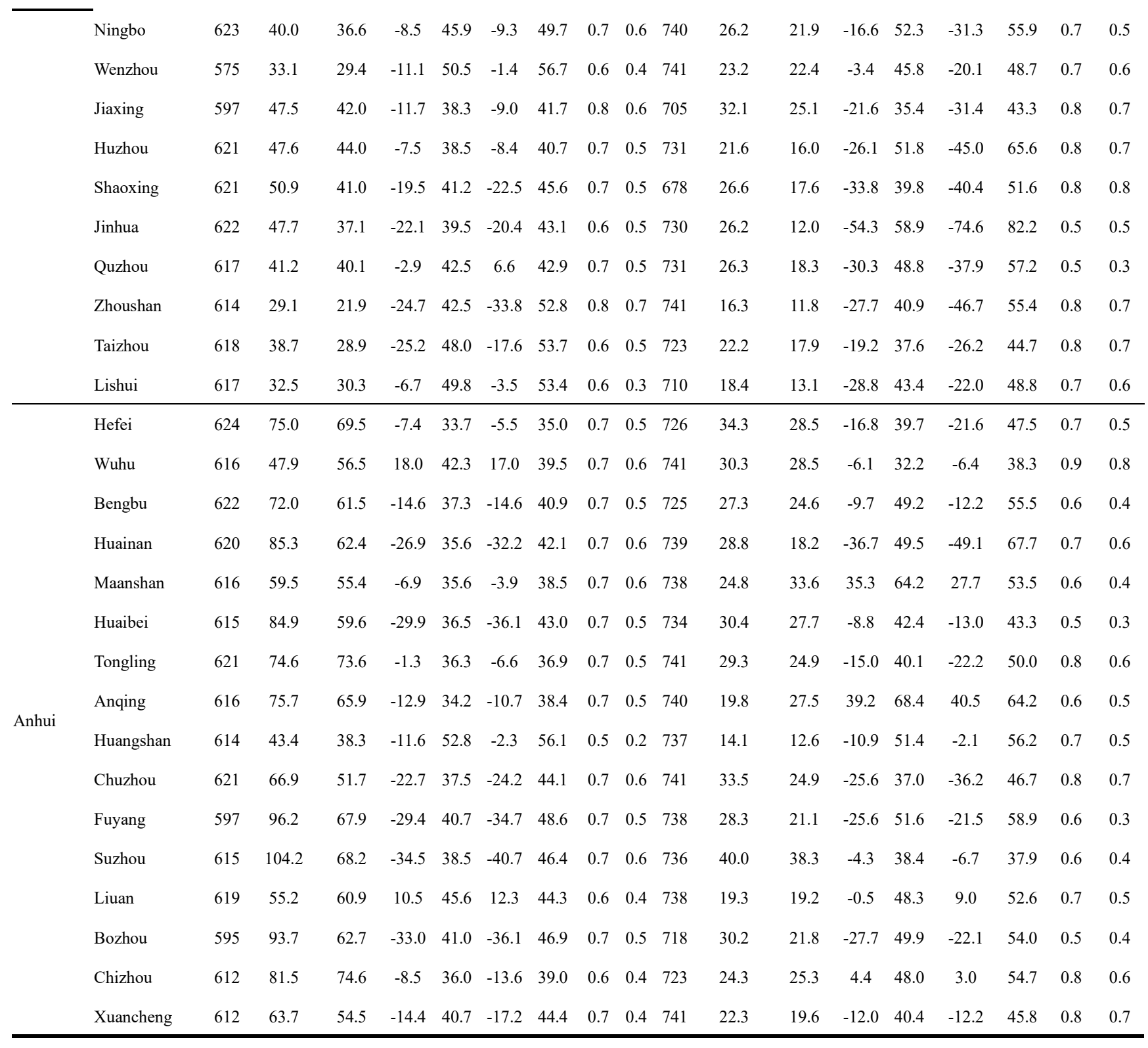

253 (e) $\mathrm{PM}_{10}$

\begin{tabular}{|c|c|c|c|c|c|c|c|c|c|c|c|c|c|c|c|c|c|c|c|}
\hline \multirow[b]{2}{*}{ Province } & \multirow[b]{2}{*}{ City } & \multicolumn{9}{|c|}{ Jan } & \multicolumn{9}{|c|}{ Jul } \\
\hline & & $\begin{array}{l}\text { Data } \\
\text { pairs }\end{array}$ & $\begin{array}{c}\text { Obs. } \\
\left(\mu \mathrm{g} / \mathrm{m}^{3}\right)\end{array}$ & $\begin{array}{c}\text { Sim. } \\
\left(\mu \mathrm{g} / \mathrm{m}^{3}\right)\end{array}$ & $\begin{array}{c}\text { NMB } \\
(\%)\end{array}$ & $\begin{array}{c}\text { NME } \\
(\%)\end{array}$ & $\begin{array}{l}\text { MFB } \\
(\%)\end{array}$ & $\begin{array}{c}\text { MFE } \\
(\%)\end{array}$ & IOA & $\mathrm{R}$ & $\begin{array}{l}\text { Data } \\
\text { pairs }\end{array}$ & $\begin{array}{l}\text { Obs. } \\
\left(\mu \mathrm{g} / \mathrm{m}^{3}\right.\end{array}$ & $\begin{array}{l}\text { Sim. } \\
\left(\mu \mathrm{g} / \mathrm{m}^{3}\right)\end{array}$ & $\begin{array}{c}\text { NMB } \\
(\%)\end{array}$ & $\begin{array}{c}\text { NME } \\
(\%)\end{array}$ & $\begin{array}{c}\text { MFB } \\
(\%)\end{array}$ & $\begin{array}{c}\text { MFE } \\
(\%)\end{array}$ & IOA & $\mathrm{R}$ \\
\hline Shanghai & Shanghai & 530 & 52.3 & 49.9 & -4.5 & 36.6 & -4.3 & 34.7 & 0.7 & 0.5 & 714 & 53.2 & 50.4 & -5.3 & 36.1 & -11.5 & 37.0 & 0.7 & 0.5 \\
\hline \multirow{8}{*}{ ngs } & Nanjing & 593 & 72.7 & 61.7 & -15.1 & 40.1 & -15.5 & 45.2 & 0.6 & 0.4 & 428 & 29.7 & 33.4 & 12.5 & 49.9 & 10.7 & 51.4 & 0.8 & 0.7 \\
\hline & Wuxi & 615 & 79.3 & 63.0 & -20.6 & 37.0 & -24.2 & 41.7 & 0.7 & 0.5 & 736 & 48.5 & 40.2 & -17.1 & 49.1 & -30.5 & 54.2 & 0.6 & 0.4 \\
\hline & Xuzhou & 566 & 147.7 & 80.9 & -45.2 & 47.6 & -60.1 & 63.1 & 0.6 & 0.5 & 696 & 64.8 & 39.3 & -39.4 & 46.2 & -49.1 & 58.3 & 0.6 & 0.5 \\
\hline & Changzhou & 612 & 82.9 & 69.0 & -16.7 & 36.1 & -17.6 & 40.4 & 0.7 & 0.5 & 702 & 44.6 & 40.5 & -9.2 & 39.0 & -21.9 & 43.2 & 0.7 & 0.6 \\
\hline & Suzhou & 430 & 64.2 & 58.3 & -9.2 & 38.0 & -4.3 & 39.0 & 0.7 & 0.5 & 611 & 53.4 & 48.6 & -9.1 & 47.9 & -18.1 & 49.0 & 0.6 & 0.4 \\
\hline & Nantong & 421 & 61.2 & 50.7 & -17.3 & 36.7 & -14.1 & 37.6 & 0.7 & 0.5 & 652 & 66.1 & 49.1 & -25.7 & 39.6 & -31.2 & 47.9 & 0.6 & 0.4 \\
\hline & Lianyungang & 581 & 85.4 & 58.0 & -32.1 & 45.6 & -34.9 & 53.5 & 0.6 & 0.5 & 632 & 51.5 & 28.3 & -44.9 & 53.1 & -57.4 & 69.8 & 0.5 & 0.2 \\
\hline & Huaian & 603 & 101.5 & 71.9 & -29.2 & 46.2 & -34.9 & 53.5 & 0.6 & 0.4 & 693 & 58.7 & 43.2 & -26.3 & 40.1 & -33.4 & 48.8 & 0.6 & 0.4 \\
\hline
\end{tabular}




\begin{tabular}{|c|c|c|c|c|c|c|c|c|c|c|c|c|c|c|c|c|c|c|c|}
\hline & Yancheng & 572 & 70.6 & 46.8 & -33.7 & 47.6 & -39.5 & 55.7 & 0.7 & 0.5 & 738 & 76.2 & 35.9 & -52.8 & 56.1 & -76.6 & 81.5 & 0.5 & 0.4 \\
\hline & Yangzhou & 578 & 92.4 & 74.9 & -18.9 & 37.7 & -20.2 & 43.9 & 0.7 & 0.5 & 675 & 64.0 & 46.1 & -28.0 & 41.8 & -38.5 & 50.8 & 0.6 & 0.5 \\
\hline & Zhenjiang & 598 & 95.9 & 59.4 & -38.1 & 42.8 & -50.5 & 55.9 & 0.6 & 0.5 & 675 & 65.6 & 39.7 & -39.4 & 48.8 & -52.8 & 63.5 & 0.6 & 0.5 \\
\hline & Taizhou & 438 & 70.0 & 59.8 & -14.7 & 41.7 & -13.7 & 43.6 & 0.7 & 0.4 & 734 & 56.0 & 49.4 & -11.8 & 40.0 & -16.7 & 43.6 & 0.6 & 0.4 \\
\hline & Suqian & 541 & 75.4 & 64.3 & -14.7 & 39.8 & -16.5 & 44.0 & 0.7 & 0.5 & 64 & 73.0 & 26.1 & -64.2 & 64.5 & -85.2 & 85.8 & 0.4 & 0.1 \\
\hline \multirow{11}{*}{ Zhejiang } & Hangzhou & 624 & 74.5 & 62.1 & -16.6 & 37.9 & -18.6 & 41.6 & 0.7 & 0.5 & 738 & 42.8 & 34.2 & -20.1 & 32.1 & -26.9 & 38.4 & 0.8 & 0.7 \\
\hline & Ningbo & 622 & 55.4 & 51.9 & -6.4 & 41.9 & -12.7 & 43.6 & 0.7 & 0.5 & 736 & 43.6 & 40.4 & -7.4 & 37.8 & -14.2 & 38.6 & 0.8 & 0.6 \\
\hline & Wenzhou & 573 & 66.0 & 44.4 & -32.7 & 48.4 & -35.2 & 58.7 & 0.5 & 0.4 & 721 & 51.6 & 51.6 & -0.1 & 38.9 & -11.7 & 40.5 & 0.7 & 0.5 \\
\hline & Jiaxing & 593 & 64.1 & 60.4 & -5.8 & 34.2 & -6.3 & 35.7 & 0.7 & 0.5 & 704 & 51.6 & 43.6 & -15.4 & 33.9 & -24.5 & 40.2 & 0.8 & 0.7 \\
\hline & Huzhou & 514 & 64.5 & 61.3 & -5.0 & 38.2 & -5.9 & 40.4 & 0.7 & 0.4 & 710 & 41.8 & 25.6 & -38.7 & 59.5 & -63.6 & 79.8 & 0.6 & 0.4 \\
\hline & Shaoxing & 617 & 80.9 & 57.6 & -28.7 & 41.8 & -31.0 & 47.4 & 0.6 & 0.4 & 677 & 43.7 & 30.9 & -29.4 & 37.3 & -36.0 & 46.0 & 0.8 & 0.7 \\
\hline & Jinhua & 507 & 62.8 & 50.7 & -19.3 & 32.5 & -19.5 & 36.4 & 0.7 & 0.5 & 684 & 43.2 & 21.4 & -50.5 & 55.7 & -67.3 & 74.4 & 0.5 & 0.3 \\
\hline & Quzhou & 583 & 70.8 & 54.9 & -22.4 & 39.2 & -19.2 & 42.6 & 0.6 & 0.5 & 710 & 50.9 & 29.2 & -42.6 & 50.9 & -53.2 & 63.3 & 0.5 & 0.2 \\
\hline & Zhoushan & 612 & 44.7 & 36.4 & -18.5 & 36.6 & -19.9 & 40.6 & 0.8 & 0.7 & 741 & 30.1 & 40.7 & 35.2 & 44.8 & 21.7 & 36.7 & 0.7 & 0.8 \\
\hline & Taizhou & 613 & 52.3 & 43.0 & -17.8 & 40.0 & -12.4 & 43.7 & 0.7 & 0.6 & 723 & 40.4 & 44.7 & 10.9 & 33.7 & 5.5 & 32.6 & 0.8 & 0.7 \\
\hline & Lishui & 515 & 43.8 & 46.5 & 6.0 & 47.9 & 8.0 & 48.9 & 0.6 & 0.3 & 651 & 38.9 & 28.1 & -27.9 & 48.3 & -23.7 & 52.2 & 0.5 & 0.2 \\
\hline \multirow{16}{*}{ Anhui } & Hefei & 389 & 94.2 & 93.4 & -0.8 & 35.9 & -0.8 & 34.2 & 0.6 & 0.4 & 622 & 51.2 & 48.8 & -4.7 & 35.2 & -7.0 & 37.4 & 0.8 & 0.6 \\
\hline & Wuhu & 597 & 76.6 & 76.8 & 0.4 & 37.3 & -1.1 & 37.4 & 0.7 & 0.5 & 727 & 52.0 & 46.5 & -10.6 & 31.7 & -9.7 & 36.2 & 0.8 & 0.7 \\
\hline & Bengbu & 604 & 104.8 & 87.1 & -16.8 & 41.1 & -18.6 & 44.8 & 0.6 & 0.4 & 721 & 51.1 & 38.1 & -25.4 & 49.5 & -29.9 & 59.2 & 0.6 & 0.4 \\
\hline & Huainan & 573 & 109.4 & 77.9 & -28.8 & 38.9 & -37.7 & 47.5 & 0.6 & 0.5 & 736 & 68.6 & 26.1 & -61.9 & 63.3 & -95.3 & 97.1 & 0.5 & 0.5 \\
\hline & Maanshan & 536 & 81.3 & 76.0 & -6.5 & 38.5 & -6.9 & 40.7 & 0.6 & 0.4 & 699 & 48.9 & 53.8 & 10.1 & 40.4 & 7.5 & 40.3 & 0.7 & 0.6 \\
\hline & Huaibei & 578 & 127.6 & 74.4 & -41.7 & 44.1 & -54.1 & 56.7 & 0.6 & 0.4 & 669 & 60.3 & 37.4 & -38.0 & 47.3 & -46.3 & 57.7 & 0.5 & 0.3 \\
\hline & Tongling & 605 & 100.5 & 96.5 & -4.0 & 36.6 & -9.4 & 37.2 & 0.6 & 0.4 & 668 & 45.1 & 35.8 & -20.7 & 40.2 & -33.0 & 50.4 & 0.7 & 0.6 \\
\hline & Anqing & 566 & 98.9 & 88.5 & -10.5 & 34.8 & -10.9 & 38.5 & 0.7 & 0.4 & 730 & 43.1 & 42.7 & -0.9 & 43.0 & -8.2 & 43.8 & 0.6 & 0.5 \\
\hline & Huangshan & 509 & 55.8 & 52.8 & -5.4 & 50.1 & -3.3 & 49.7 & 0.4 & 0.0 & 735 & 43.3 & 23.5 & -45.8 & 51.6 & -65.4 & 70.9 & 0.5 & 0.4 \\
\hline & Chuzhou & 599 & 105.9 & 74.3 & -29.9 & 40.2 & -35.4 & 48.5 & 0.6 & 0.4 & 683 & 54.0 & 43.0 & -20.5 & 35.5 & -27.2 & 42.9 & 0.8 & 0.6 \\
\hline & Fuyang & 564 & 148.3 & 87.2 & -41.2 & 46.9 & -53.6 & 60.5 & 0.6 & 0.4 & 733 & 60.1 & 30.3 & -49.5 & 55.7 & -62.1 & 70.0 & 0.5 & 0.3 \\
\hline & Suzhou & 497 & 145.6 & 84.5 & -42.0 & 44.0 & -52.6 & 55.2 & 0.6 & 0.4 & 637 & 53.7 & 52.9 & -1.7 & 34.6 & -5.4 & 33.0 & 0.6 & 0.4 \\
\hline & Liuan & 507 & 77.5 & 81.2 & 4.8 & 45.8 & 5.9 & 45.3 & 0.5 & 0.3 & 729 & 47.0 & 31.2 & -33.7 & 45.0 & -36.6 & 51.1 & 0.5 & 0.4 \\
\hline & Bozhou & 550 & 133.0 & 78.2 & -41.2 & 47.0 & -52.8 & 59.3 & 0.5 & 0.3 & 696 & 63.0 & 31.0 & -50.9 & 56.6 & -55.5 & 66.3 & 0.4 & 0.4 \\
\hline & Chizhou & 561 & 118.6 & 108.7 & -8.4 & 38.0 & -12.3 & 39.7 & 0.6 & 0.3 & 731 & 55.4 & 50.0 & -9.9 & 42.1 & -19.0 & 48.6 & 0.7 & 0.5 \\
\hline & Xuancheng & 507 & 88.0 & 75.1 & -14.7 & 40.2 & -18.0 & 43.5 & 0.6 & 0.3 & 728 & 41.9 & 36.4 & -13.3 & 42.1 & -14.9 & 46.0 & 0.7 & 0.6 \\
\hline 254 & (f) $\mathrm{CO}$ & & & & & & & & & & & & & & & & & & \\
\hline
\end{tabular}

Jan

Jul

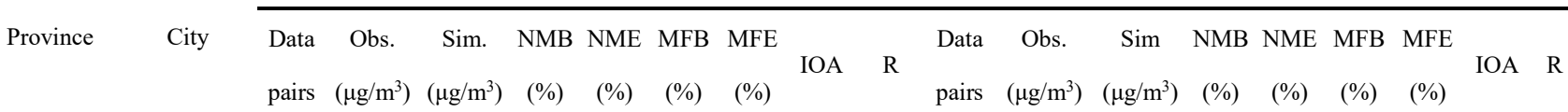

\begin{tabular}{|c|c|c|c|c|c|c|c|c|c|c|c|c|c|c|c|c|c|c|c|}
\hline Shanghai & Shanghai & 624 & 0.9 & 0.6 & -35.6 & 38.6 & -42.4 & 46.1 & 0.7 & 0.7 & 741 & 0.5 & 0.4 & -22.0 & 36.1 & -31.2 & 42.5 & 0.6 & 0.4 \\
\hline \multirow{2}{*}{ Jiangsu } & Nanjing & 606 & 1.0 & 0.7 & -26.2 & 36.3 & -26.4 & 41.3 & 0.6 & 0.4 & 709 & 1.0 & 0.4 & -62.4 & 63.3 & -90.4 & 92.4 & 0.4 & 0.2 \\
\hline & Wuxi & 618 & 1.1 & 0.8 & -30.0 & 37.2 & -33.1 & 41.8 & 0.6 & 0.4 & 740 & 0.7 & 0.4 & -44.3 & 48.3 & -60.2 & 64.0 & 0.4 & 0.1 \\
\hline
\end{tabular}




\begin{tabular}{|c|c|c|c|c|c|c|c|c|c|c|c|c|c|c|c|c|c|c|c|}
\hline & Xuzhou & 581 & 1.3 & 1.0 & -21.7 & 35.1 & -27.2 & 38.5 & 0.6 & 0.5 & 739 & 0.8 & 0.6 & -24.5 & 48.3 & -27.8 & 52.0 & 0.4 & 0.1 \\
\hline & Changzhou & 621 & 0.9 & 0.9 & -4.2 & 37.3 & -1.0 & 38.1 & 0.6 & 0.3 & 701 & 0.7 & 0.5 & -32.6 & 40.9 & -36.6 & 46.2 & 0.5 & 0.3 \\
\hline & Suzhou & 623 & 0.9 & 0.8 & -17.0 & 28.2 & -17.6 & 29.5 & 0.7 & 0.6 & 618 & 0.4 & 0.5 & 9.6 & 38.3 & 9.0 & 35.4 & 0.6 & 0.3 \\
\hline & Nantong & 619 & 0.9 & 0.8 & -9.9 & 27.8 & -12.0 & 28.4 & 0.7 & 0.5 & 680 & 0.8 & 0.6 & -27.5 & 38.1 & -33.9 & 44.1 & 0.5 & 0.2 \\
\hline & Lianyungang & 596 & 1.1 & 0.6 & -39.4 & 43.4 & -45.5 & 51.3 & 0.6 & 0.5 & 741 & 0.5 & 0.3 & -30.4 & 38.1 & -30.3 & 41.3 & 0.5 & 0.3 \\
\hline & Huaian & 617 & 0.9 & 1.0 & 7.1 & 39.2 & 5.9 & 36.2 & 0.6 & 0.4 & 740 & 0.7 & 0.6 & -7.5 & 36.2 & -4.2 & 35.7 & 0.5 & 0.1 \\
\hline & Yancheng & 617 & 0.7 & 0.6 & -8.8 & 29.9 & -6.4 & 29.0 & 0.7 & 0.6 & 739 & 0.9 & 0.4 & -51.8 & 52.2 & -72.0 & 72.4 & 0.4 & 0.3 \\
\hline & Yangzhou & 624 & 1.0 & 0.8 & -21.6 & 29.9 & -24.3 & 32.9 & 0.7 & 0.6 & 689 & 0.6 & 0.5 & -24.2 & 36.0 & -27.0 & 40.1 & 0.5 & 0.2 \\
\hline & Zhenjiang & 621 & 1.0 & 0.9 & -6.8 & 28.6 & -10.9 & 29.4 & 0.7 & 0.5 & 741 & 0.9 & 0.6 & -29.0 & 37.1 & -35.4 & 43.8 & 0.5 & 0.2 \\
\hline & Taizhou & 500 & 1.0 & 0.7 & -32.3 & 38.4 & -38.3 & 44.7 & 0.6 & 0.4 & 736 & 0.7 & 0.5 & -19.2 & 38.8 & -21.3 & 40.3 & 0.4 & 0.1 \\
\hline & Suqian & 619 & 1.2 & 0.7 & -41.2 & 42.5 & -54.7 & 55.8 & 0.6 & 0.6 & 66 & 0.4 & 0.3 & -18.5 & 24.4 & -19.3 & 25.6 & 0.5 & 0.2 \\
\hline \multirow{11}{*}{ Zhejiang } & Hangzhou & 624 & 1.0 & 0.7 & -29.4 & 33.0 & -34.5 & 38.5 & 0.6 & 0.5 & 740 & 0.7 & 0.4 & -47.4 & 53.8 & -61.7 & 69.8 & 0.4 & -0.1 \\
\hline & Ningbo & 623 & 0.8 & 0.6 & -18.1 & 36.4 & -24.1 & 39.5 & 0.6 & 0.4 & 740 & 0.6 & 0.3 & -47.4 & 51.6 & -65.9 & 69.6 & 0.5 & 0.3 \\
\hline & Wenzhou & 575 & 0.7 & 0.6 & -12.1 & 27.8 & -10.5 & 29.2 & 0.7 & 0.5 & 741 & 0.6 & 0.4 & -36.1 & 42.7 & -48.3 & 54.4 & 0.6 & 0.4 \\
\hline & Jiaxing & 597 & 0.8 & 0.7 & -21.0 & 40.9 & -13.2 & 42.2 & 0.6 & 0.4 & 705 & 0.7 & 0.4 & -34.2 & 40.2 & -46.8 & 51.9 & 0.5 & 0.4 \\
\hline & Huzhou & 620 & 1.0 & 0.6 & -36.9 & 37.7 & -46.5 & 47.3 & 0.6 & 0.6 & 722 & 0.6 & 0.3 & -55.4 & 55.6 & -80.3 & 80.5 & 0.4 & 0.5 \\
\hline & Shaoxing & 623 & 0.9 & 0.7 & -19.0 & 31.2 & -19.1 & 33.0 & 0.7 & 0.5 & 679 & 0.7 & 0.3 & -54.7 & 56.1 & -78.5 & 79.8 & 0.4 & 0.4 \\
\hline & Jinhua & 623 & 0.9 & 0.6 & -35.0 & 36.3 & -42.7 & 44.3 & 0.6 & 0.5 & 734 & 0.6 & 0.2 & -60.8 & 61.0 & -86.2 & 86.5 & 0.4 & 0.4 \\
\hline & Quzhou & 623 & 1.0 & 0.8 & -11.8 & 36.5 & -17.9 & 37.4 & 0.5 & 0.2 & 727 & 0.5 & 0.6 & 21.4 & 61.8 & 13.2 & 52.6 & 0.4 & 0.2 \\
\hline & Zhoushan & 614 & 0.8 & 0.4 & -51.2 & 51.5 & -71.3 & 71.6 & 0.5 & 0.7 & 727 & 0.4 & 0.2 & -56.9 & 57.1 & -81.1 & 81.3 & 0.4 & 0.5 \\
\hline & Taizhou & 622 & 0.8 & 0.5 & -38.0 & 40.7 & -42.9 & 46.1 & 0.5 & 0.5 & 741 & 0.6 & 0.2 & -58.1 & 58.1 & -84.3 & 84.3 & 0.4 & 0.7 \\
\hline & Lishui & 621 & 0.5 & 0.4 & -17.0 & 27.3 & -20.1 & 30.8 & 0.7 & 0.5 & 712 & 0.5 & 0.2 & -63.6 & 63.6 & -93.3 & 93.3 & 0.4 & 0.7 \\
\hline \multirow{16}{*}{ Anhui } & Hefei & 618 & 1.0 & 0.8 & -26.4 & 32.2 & -27.8 & 35.3 & 0.7 & 0.6 & 732 & 0.7 & 0.4 & -50.0 & 50.2 & -66.8 & 67.1 & 0.4 & 0.5 \\
\hline & Wuhu & 614 & 1.1 & 0.7 & -33.2 & 40.0 & -41.6 & 49.0 & 0.5 & 0.3 & 725 & 0.9 & 0.4 & -56.9 & 57.8 & -76.3 & 77.6 & 0.5 & 0.4 \\
\hline & Bengbu & 621 & 1.0 & 0.7 & -24.1 & 34.7 & -30.9 & 38.8 & 0.7 & 0.5 & 739 & 0.6 & 0.3 & -39.7 & 41.2 & -48.4 & 50.1 & 0.5 & 0.4 \\
\hline & Huainan & 615 & 1.3 & 0.8 & -35.4 & 38.6 & -44.1 & 47.3 & 0.6 & 0.4 & 740 & 0.8 & 0.3 & -63.1 & 64.1 & -94.3 & 95.7 & 0.4 & 0.4 \\
\hline & Maanshan & 591 & 1.0 & 0.9 & -7.7 & 36.5 & -5.3 & 35.9 & 0.5 & 0.2 & 732 & 0.8 & 0.5 & -46.1 & 50.6 & -54.2 & 62.5 & 0.5 & 0.3 \\
\hline & Huaibei & 596 & 1.4 & 1.7 & 22.0 & 52.6 & 6.8 & 41.7 & 0.4 & 0.3 & 737 & 0.8 & 1.4 & 81.7 & 89.2 & 50.0 & 57.3 & 0.3 & 0.2 \\
\hline & Tongling & 604 & 0.9 & 0.8 & -9.5 & 41.3 & -0.3 & 45.3 & 0.6 & 0.4 & 736 & 0.7 & 0.4 & -47.2 & 54.1 & -50.9 & 65.3 & 0.5 & 0.4 \\
\hline & Anqing & 620 & 0.8 & 0.9 & 7.3 & 30.2 & 7.0 & 28.5 & 0.6 & 0.4 & 739 & 0.5 & 0.5 & 1.5 & 39.5 & -5.0 & 33.8 & 0.4 & 0.3 \\
\hline & Huangshan & 619 & 0.7 & 0.5 & -22.9 & 29.1 & -27.3 & 33.4 & 0.7 & 0.6 & 739 & 0.7 & 0.2 & -71.0 & 71.0 & -108.8 & 108.8 & 0.3 & -0.1 \\
\hline & Chuzhou & 620 & 0.9 & 0.7 & -25.6 & 31.3 & -29.7 & 35.3 & 0.7 & 0.6 & 741 & 0.5 & 0.3 & -30.1 & 38.5 & -34.2 & 43.8 & 0.6 & 0.4 \\
\hline & Fuyang & 587 & 1.1 & 0.8 & -33.3 & 41.2 & -37.0 & 48.4 & 0.6 & 0.5 & 736 & 0.5 & 0.3 & -33.0 & 40.9 & -25.4 & 40.5 & 0.5 & 0.6 \\
\hline & Suzhou & 611 & 1.0 & 0.9 & -9.6 & 44.9 & -18.5 & 41.7 & 0.6 & 0.5 & 735 & 0.6 & 0.3 & -42.6 & 43.4 & -52.8 & 53.5 & 0.5 & 0.5 \\
\hline & Liuan & 617 & 0.9 & 0.6 & -27.1 & 35.6 & -27.7 & 39.0 & 0.6 & 0.5 & 735 & 0.4 & 0.2 & -30.9 & 36.0 & -30.8 & 39.1 & 0.5 & 0.4 \\
\hline & Bozhou & 598 & 1.3 & 0.8 & -35.5 & 39.3 & -44.1 & 48.1 & 0.6 & 0.5 & 723 & 0.9 & 0.3 & -65.3 & 65.3 & -95.2 & 95.2 & 0.4 & 0.5 \\
\hline & Chizhou & 622 & 0.9 & 0.7 & -24.6 & 33.5 & -28.2 & 37.3 & 0.5 & 0.3 & 738 & 0.5 & 0.2 & -54.7 & 55.2 & -70.6 & 71.4 & 0.5 & 0.5 \\
\hline & Xuancheng & 612 & 0.8 & 0.6 & -19.1 & 27.8 & -21.9 & 30.3 & 0.7 & 0.6 & 741 & 0.7 & 0.2 & -65.5 & 65.5 & -97.7 & 97.7 & 0.3 & 0.5 \\
\hline
\end{tabular}


SO-This study



$\mathrm{SO}_{2}$-MEIC 2016

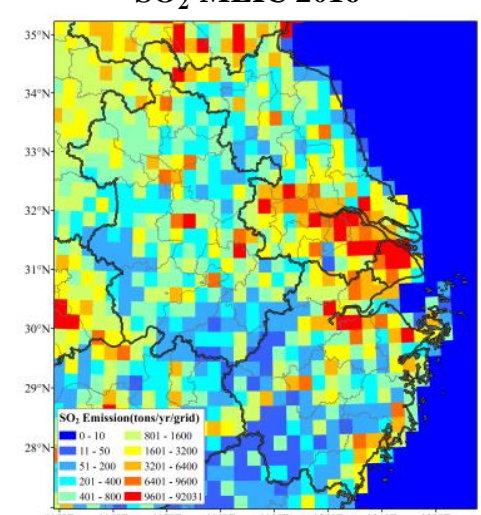

NOx-This study

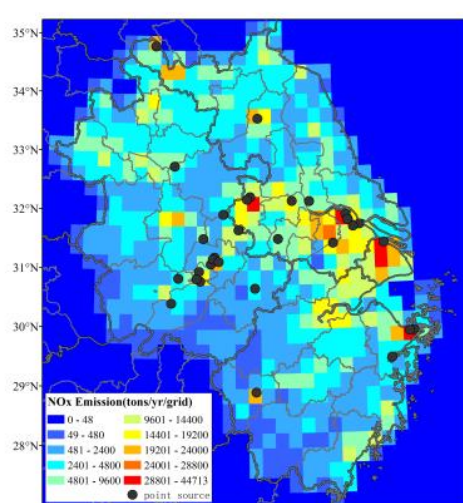

NOx-MEIC 2016

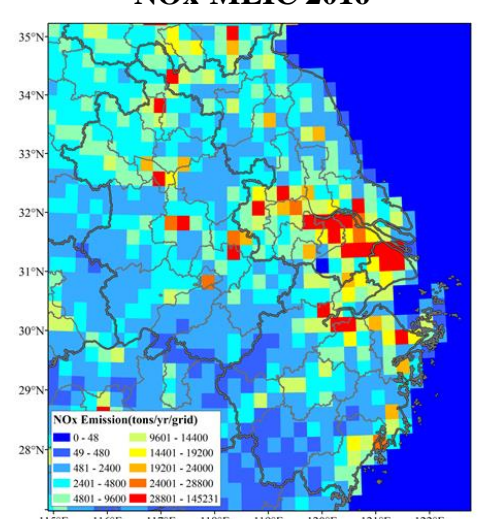

NMVOCs-This study

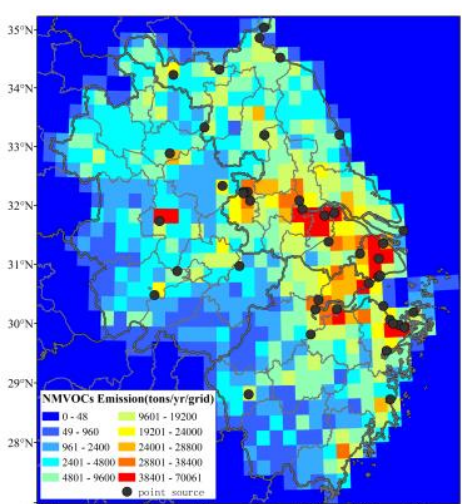

NMVOCs-MEIC 2016

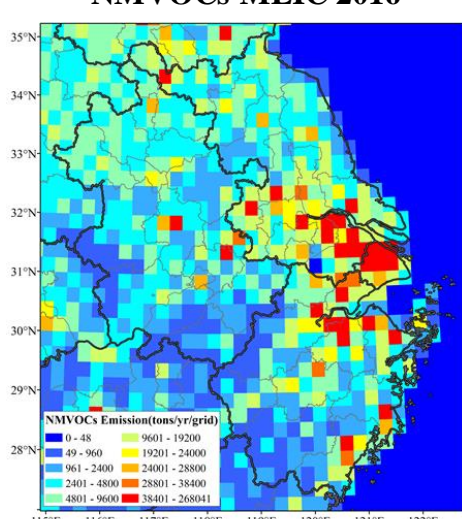

$\mathrm{NH}_{3}$-This study



$\mathrm{NH}_{3}$-MEIC 2016

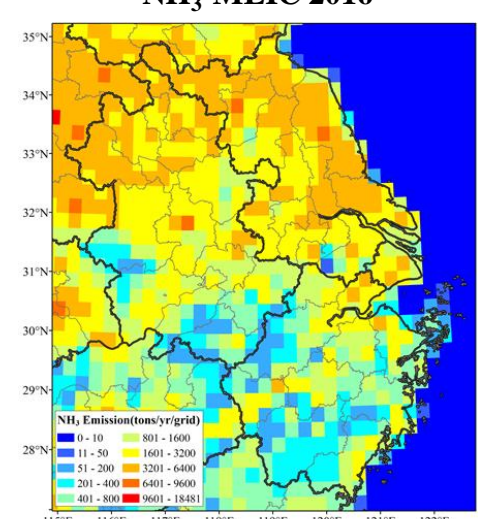

$\mathbf{P M}_{2.5}$-This study

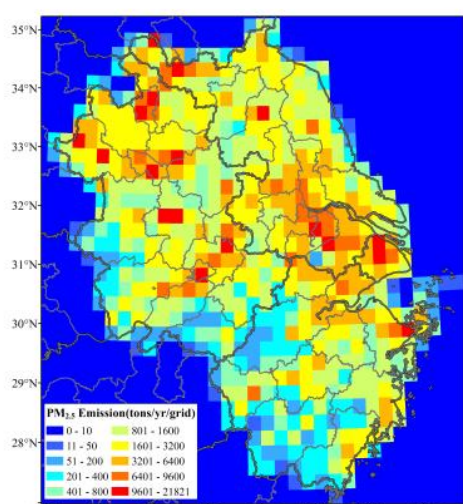

PM $_{2,5}$-MEIC 2016

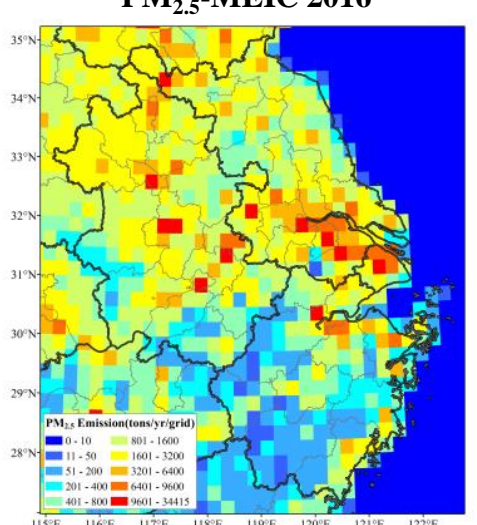

Figure S1. Comparisons of spatial distributions of $\mathrm{SO}_{2}, \mathrm{NO}_{x}, \mathrm{NMVOCs}, \mathrm{NH}_{3}$, and $\mathrm{PM}_{2.5}$ emissions between this study and the MEIC. The black dots represent for large point sources. 

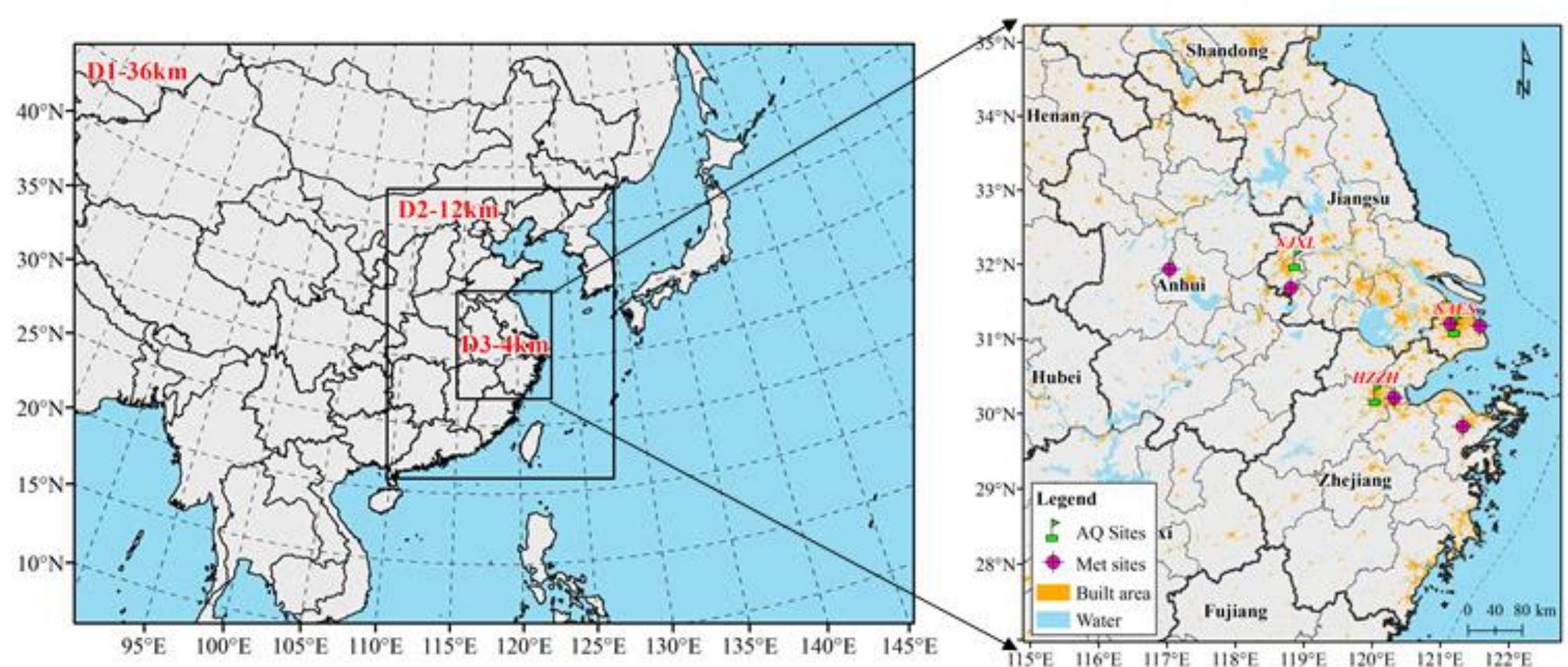

261 Figure S2. Nested Modeling domain (left) and observation sites (right) for model validation. 


\section{References}

264 Chen, Y.: Study on characteristics ammonia emission from typical large scale pig farm in Shanghai, Master Thesis, East China University of Science and Technology, 2017a (in Chinese).

Chen, Y., Fang, X. F., Shen, G. X., Xu, C., Qian, X. Y., Zhao, Z. G., Yu, S. F., Li, J. W., and Wang, Z. Q.: Research on ammonia emission characteristics and its influencing factors from paddy fields in Taihu Lake region, Acta Agriculturae Zhejiangensis, 29, 119-128, $2017 b$ (in Chinese).

European Environment Agency (EEA): EMEP/EEA air pollution emission inventory guidebook, $\quad$ http://www.eea.europa.eu/publications/emep-eea-emission-inventoryguidebook-2013, 2013.

Fan, Q. Z., Zhang, Y., Ma, W. C., Ma, H. X., Feng, J. L., Yu, Q., Yang, X., Ng, S. K. W., Fu, Q. Y., and Chen, L. M.: Spatial and seasonal dynamics of ship emissions over the Yangtze River Delta and East China Sea and their potential environmental influence, Environ. Sci. Technol., 50, 1322-1329, 2016.

281 Fu, M. L., Ding, Y., Yin, H., Ji, Z., Ge, Y. S., and Liang, B.: Characteristics of agricultural tractors emissions under real-world operating cycle, Transactions of the Chinese Society

Gao, Y. Q., Wang, H. L., Xu, R. Z., Jing, S. A., Liu, Y. H., and Peng, Y. R.: Characterization of volatile organic compounds from cooking emissions, Huanjing Kexue, 40, 1627-1633, 2019 (in Chinese).

Ge, Y. S., Liu, H. K., Ding, Y., Yin, H., Fu, M. L., and Li, J. Q.: Experimental study on characteristics of emissions and fuel consumption for combines, Transactions of the Chinese Society of Agricultural Engineering, 29, 41-47, 2013 (in Chinese).

Guo, Y. Y., Gao, X., Zhu, T. Y., Luo, L., and Zheng, Y.: Chemical profiles of PM emitted from 
the iron and steel industry in northern China, Atmos. Environ., 150, 187-197, 2017.

Huang, C., Wang, H. L., Li, L., Wang, Q., Lu, Q., de Gouw, J. A., Zhou, M., Jing, S. A., Lu, J., and Chen, C. H.: VOC species and emission inventory from vehicles and their SOA formation potentials estimation in Shanghai, China, Atmos. Chem. Phys., 15, 1108111096, 2015.

Huang, C., Lou, S. R., Qiao, L. P., Jing, S. A., Tao, S. K., Zhou, M., and Chen, C. H.: Physicochemical characteristics of real-world PM emissions from heavy-duty diesel buses, Research of Environmental Sciences, 29, 1352-1361, 2016 (in Chinese).

Huang, C., Tao, S. K., Lou, S. R., Hu, Q. Y., Wang, H. L., Wang, Q., Li, L., Wang, H. Y., Liu, J. G., Quan, Y. F., and Zhou, L. L.: Evaluation of emission factors for light-duty gasoline vehicles based on chassis dynamometer and tunnel studies in Shanghai, China, Atmos. Environ., 169, 193-203, 2017.

Huang, C., Hu, Q. Y., and Lu, J.: Measurements of OC and EC emission factors for light-duty gasoline vehicles, Huanjing Kexue, 39, 3110-3117, 2018a (in Chinese).

Huang, C., Hu, Q. Y., Lou, S. R., Tian, J. J., Wang, R. N., Xu, C., An, J. Y., Ren, H. J., Ma, D., Quan, Y. F., Zhang, Y. J., and Li, L.: Ammonia emission measurements for light-duty gasoline vehicles in China and implications for emission modeling, Environ. Sci. Technol., $52,11223-11231,2018 b$.

Huang, C., Hu, Q. Y., Wang, H. Y., Qiao, L. P., Jing, S. A., Wang, H. L., Zhou, M., Zhu, S. H., Ma, Y. G., Lou, S. R., Li, L., Tao, S. K., Li, Y. J., and Lou, D. M.: Emission factors of particulate and gaseous compounds from a large cargo vessel operated under real-world conditions, Environ. Pollut., 242, 667-674, 2018c.

Hsu, Y., Divita, F., and Dorn, J.: SPECIATE version 4.4 database development documentation, final report, EPA/600/R-13/307, 2014.

International Civil Aviation Organization (ICAO): ICAO Environmental Report 2013, Montreal, Canada, 2014.

Jing, S. A., Wang, H. L., Zhu, H. L., Yang, Q., Lu, B., Xia, Y., Yu, C. G., Tao, S. K., Li, L., Lou, S. R., Huang, C., Tang, W., and Jing, B. L.: Treatment status and emission characteristics 
of volatile organic compounds from typical industrial sources, Huanjing Kexue, 39, 30913095, 2018 (in Chinese).

321 Li, M., Zhang, Q., Kurokawa, J. i., Woo, J. H., He, K., Lu, Z., Ohara, T., Song, Y., Streets, D. G., Carmichael, G. R., Cheng, Y., Hong, C., Huo, H., Jiang, X., Kang, S., Liu, F., Su, H., Zheng, B.: MIX: a mosaic Asian anthropogenic emission inventory under the international collaboration framework of the MICS-Asia and HTAP. Atmos. Chem. Phys., 17, 935-963, 2017.

Li, Z., Zhang, K. S., Pang, K. L., and Di, B. F.: A fuel-based approach for emission factor development for highway paving construction equipment in China, J. Air Waste Manage. Assoc., 66, 1214-1223, 2016.

Liu, Z. Q.: Study on the estimation of $\mathrm{NO}_{x}$ from thermal power industry in China, Master thesis, North China Electric Power University, China, Beijing, 2008 (in Chinese).

Lou, S. R.: Emission characteristics of $\mathrm{PM}_{2.5}$ from medium and small capacity coal-fired boilers in service, Acta Scientiae Circumstantiae, 34, 3119-3125, 2014 (in Chinese).

Lu, J., Huang, C., Hu, Q. Y., Yang, Q., Jing, B. L., Xia, Y., Lu, B., Tang, W., Lou, S. R., Tao, S. K., and Li, L.: Air pollutant emissions inventory of non-road machinery in typical cities in Eastern China, Huanjing Kexue, 38, 2738-2746, 2017 (in Chinese).

Ministry of Environmental Protection (MEP), P.R. of China: Guidebooks for compiling air pollution emission inventories. Available via. http://www.zhb.gov.cn/gkml/hbb/bgg/ 201408/t20140828_288364.htm, 2014 (in Chinese).

Qu, L., He, L. Q., Hu, J. N., Jin, T. S., Zu, L., Wang, M. Y., and Song, J. H.: Characteristics of $\mathrm{PM}_{2.5}$ emissions and its carbonaceous components from construction machines under

343 Sun, Y. Y.: Study on emission inventory and uncertainty assessment of multi-pollutants from coal-fired power plants, Master thesis, Zhejiang University, China, Hangzhou, 2015 (in different typical driving modes, Research of Environmental Sciences, 28, 1047-1052, 2015 (in Chinese). Chinese).

Tang, W., Yang, Q., Huang, C., Lu, B., Xia, Y., Jing, B. L., Lu, Q., and Lu, J.: Study on 
characteristics of pollutant emission from motor vehicles in Hangzhou based on large data analysis and IVE model, Acta Scientiae Circumstantiae, 38, 71-78, 2018 (in Chinese).

U.S. Environmental Protection Agency (USEPA): Compilation of air pollutant emission Factors, AP-42, 5th ed., Washington, DC, 2002.

U.S. Environmental Protection Agency (USEPA): Exhaust and crankcase emission factors for non-road engine modeling-compression ignition, Washington, DC, 2010.

U.S. Environmental Protection Agency (USEPA): Emissions estimation protocol for petroleum refineries, Washington, DC, 2015.

Wang, S., Zhu, F. H., Wang, H. M., Zuo, Y., Sun, X. L., Zhao, X. Y., Chen, H., and Liu, G.: Fine particle emission characteristics from coal-fired power plants based on field test, Acta Scientiae Circumstantiae, 31, 630-635, 2011 (in Chinese).

Wang, H. L., Lou, S. R., Huang, C., Qiao, L. P., Tang, X. B., Chen, C. H., Zeng, L. M., Wang, Q., Zhou, M., Lu, S. H., and Xu, X. N.: Source profiles of volatile organic compounds from biomass burning in Yangtze River Delta, China, Aerosol and Air Quality Research, 14, 818-828, 2014a.

Wang, H. L., Qiao, Y. Z., Chen, C. H., Lu, J., Dai, H. X., Qiao, L. P., Lou, S. R., Huang, C., Li, compounds from solvent use in Shanghai, China, Aerosol and Air Quality Research, 14, $301-310,2014 b$.

Wang, H. L., Jing, S. A., Wang, Q., Huang, C., Lou, S. R., Qiao, L. P., and Li, L.: Measurement and characterization of emissions of volatile organic compounds in solvent use, Research of Environmental Sciences, 29, 1433-1439, 2016 (in Chinese).

Wang, H. L., Yang, Z. X., and Jing, S. A.: Volatile organic compounds ( VOCs) source profiles of industrial processing and solvent use emissions: a review, Huanjing Kexue, 38, $2617-$ 2628, 2017a (in Chinese).

Wang, H. L., Jing, S. A., Lou, S. R., Hu, Q. Y., Li, L., Tao, S. K., Huang, C., Qiao, L. P., and Chen, C. H.: Volatile organic compounds (VOCs) source profiles of on-road vehicle emissions in China, Sci. Total Environ., 607-608, 253-261, $2017 \mathrm{~b}$. 
Wang, H. L., Jing, S. A., Lou, S. R., Tao, S. K., Qiao, L. P., Li, L., Huang, C., Lin, L., and Chen, C. H.: Estimation of fine particle $\left(\mathrm{PM}_{2.5}\right)$ emission inventory from cooking: case study for Shanghai, Huanjing Kexue, 39, 1971-1977, 2018 (in Chinese).

Wayson, R. L., Fleming, G. G., and Lovinelli, R.: Methodology to estimate particulate matter emissions from certified commercial aircraft engines, J. Air Waste Manage, 59, 91-100, 2009.

Xia, Y., Yang, Q., Xu, C., Lu, B., Tang, W., Jing, B. L., Lu, Q., Huang, C., and Lu, J.: Study on high resolution $\mathrm{NH}_{3}$ emission inventory based on analysis of large data for agriculture in Hangzhou, Acta Scientiae Circumstantiae, 38, 661-668, 2018 (in Chinese).

Xu, J., Huang, C., Li, L., Chen, Y. H., Lou, S. R., Qiao, L. P., and Wang, H. Y.: Chemical composition characteristics of $\mathrm{PM}_{2.5}$ emitted by medium and small capacity coal-fired boilers in the Yangtze River Delta Region, Huanjing Kexue, 39, 1493-1501, 2018 (in Chinese).

Tang, X. B., Huang, C., Lou, S. R., Qiao, L. P., Wang, H. L., Zhou, M., Chen, M. H., Chen, C. H., Wang, Q., Li, G. L., Li, L., Huang, H. Y., and Zhang, G. F.: Emission factors and PM chemical composition study of biomass burning in the Yangtze River Delta Region, Huanjing Kexue, 35, 1623-1632, 2014 (in Chinese).

Yao, Z. M., Teng, Y., Li, J., Wu, X. F., and Duan, N.: $\mathrm{NO}_{x}$ emission factors from medium and small layer burning boilers firing soft coal, Research of Environmental Sciences, 22, 1263-1268, 2009 (in Chinese).

Zheng, M., Zhang, Y. J., Yan, C. Q., Fu, H. Y., Niu, H. Y., Huang, K., Hu, M., Zeng, L. M., Liu, Q. Z., Pei, B., and Fu, Q. Y.: Establishing $\mathrm{PM}_{2.5}$ industrial source profiles in Shanghai, China Environmental Science, 33, 1354-1359, 2013 (in Chinese).

Zhao, Y., Wang, S. X., Nielsen, C. P., Li, X. H., and Hao, J. M.: Establishment of a database of emission factors for atmospheric pollutants from Chinese coal-fired power plants, Atmos. Environ., 44, 1515-1523, 2010.

Zhou, Z. Q.: Study on characteristics ammonia emission from typical large scale dairy cow 402 farm in Shanghai, Master Thesis, East China University of Science and Technology, 2019 
403 (in Chinese).

404

405

406 